\title{
FAMÍLIA DE RETIFICADORES MULTIPULSOS COM TOPOLOGIAS DIFERENCIAIS DE TRANSFORMADORES
}

\author{
Priscila da Silva Oliveira* \\ Rodolfo Castanho Fernandes* \\ Falcondes José Mendes de Seixas * \\ falcon@dee.feis.unesp.br \\ *UNESP - Univ. Estadual Paulista - Departamento de Engenharia Elétrica \\ Campus de Ilha Solteira - SP, Brasil
}

\section{RESUMO}

Retificadores multipulsos, obtidos a partir de conexões especiais de autotransformadores, ou conexões diferenciais, estão em crescente desenvolvimento para equipar o estágio de entrada de conversores estáticos. Essas topologias retificadoras são cada vez mais atrativas não só pela robustez, como pela característica em mitigar harmônicos importantes na corrente da linha, reduzindo a distorção harmônica total de corrente (DHTi) e elevando o fator de potência. Diferentemente das conexões defasadoras isoladas (delta-estrela, ziguezague, etc.), quando se trata de autotransformador, a maior parte da energia requerida pela carga é diretamente conduzida através dos enrolamentos e apenas uma pequena fração é processada pelo núcleo, aumentando assim a densidade de potência do conversor. Este trabalho apresenta um método matemático simples baseado em diagramas fasoriais, que resulta em expressões capazes de definir todas as conexões diferenciais para as topologias Estrela e Delta, tanto elevadoras como abaixadoras de tensão, em retificadores de 12 ou 18 pulsos. As expressões obtidas, também apresentadas sob a forma de ábacos possibilitam que, para qualquer tensão da rede, seja escolhida uma determinada tensão de saída, ou vice-versa. Este fato mostra que, diferentemente das conexões defasadoras de tensão usuais, a família de conversores proposta pode ser projetada para qualquer

Artigo submetido em 03/05/2010 (Id.: 01140)

Revisado em 18/09/2010

Aceito sob recomendação do Editor Associado Prof. Takashi Yoneyama relação entre as tensões da rede de entrada e da carga. Uma aplicação imediata seria o "retrofit", ou seja, substituir um sistema retificador trifásico convencional, com baixa qualidade da energia processada, pelo retificador de 12 ou 18 pulsos, com conexões Estrela ou Deltas-diferenciais, mantendo os níveis originais de tensão da rede e da carga. O procedimento de projeto, simples e rápido, é desenvolvido e aplicado a um protótipo de $6 \mathrm{~kW}, 18$ pulsos, do tipo Delta-diferencial, com tensão média retificada de $400 \mathrm{~V}$ na carga.

PALAVRAS-CHAVE: conexão diferencial, autotransformador, multipulsos, retificador trifásico, fator de potência.

\section{ABSTRACT}

\section{A Family of Multipulse Rectifiers with Differential Topologies of Transformers}

Multipulse rectifier topologies based on auto-connections or differential connections, are more and more applied as interface stages between the mains and power converters. These topologies are becoming increasingly attractive not only for robustness, but to mitigate many low order current harmonics in the utility, reducing the total harmonic distortion of the line currents (THDi) and increasing the power factor requirements. Unlike isolated connections (delta-wye, zigzag, etc.), when the differential transformer is employed, most of the energy required by the load is directly conducted through the windings. Thus, only a 
small fraction of the kVA is processed by the magnetic core. This feature increases the power density of the converter. This paper presents a mathematical model based on phasor diagrams, which results in a single expression able to merge all differential connections (wye and delta), for both step-up and step-down rectifiers for 12 or 18 pulses. The proposed family of converters can be designed for any relationship between the line input voltage and the DC voltage, unlike the conventional phase-shift voltage connections. An immediate application would be the "retrofit", i.e. to replace a conventional rectifier with poor quality of the processed energy by the 12 or 18-pulse rectifiers with Wye or Delta-differential connections, keeping the original values for the input and load voltages. The simple and fast design procedure is developed and tested for a prototype rating 6 $\mathrm{kW}$ and $400 \mathrm{~V}$ on DC load.

KEYWORDS: Differential connection, autotransformer, multipulse, three-phase rectifier, power factor correction.

\section{INTRODUÇÃO}

O fator qualidade de energia, em conversores aplicados na eletrônica de potência, vem sendo amplamente discutido. Existem trabalhos recentes na literatura, que buscam uma melhora na qualidade da corrente de entrada em retificadores trifásicos a diodos, com a associação de um estágio CC-CC regulador (por exemplo, os estágios Boost ou SEPIC) ao estágio retificador, resultando em conversores híbridos como é apresentado por Freitas et alli (2006), além de outros conversores trifásicos que utilizam modulação por largura de pulsos (PWM), como mostrado por Pomílio et alii (1997). Técnicas como comutação suave e estratégias de controle digital também são relatadas por Freitas et alii (2006) e Kazmierkowski et alii(2009), a fim de se obter baixas perdas e elevado Fator de Potência (FP) no estágio CA-CC. Os conversores $\mathrm{CA}-\mathrm{CC}$ podem ser utilizados nas mais diversas aplicações, incluindo aproveitamentos de energia renovável, como o condicionamento de energia em turbinas eólicas, apresentado por Chen et alli (2009). Os retificadores com controle do ângulo de disparo, utilizando tiristores em sua topologia retificadora, pertencem à outra classe de conversores CA-CC e são mais adequados a elevadas potências, onde as correntes estão na faixa de quiloamperes.

A aplicação de conversores multipulsos trifásicos para correção do fator de potência é uma técnica bem aceita como discute Paice (1996), pois promove baixa ondulação na tensão de saída e uma redução na distorção harmônica total de corrente de entrada. Nos últimos anos, muitos trabalhos vêm propondo novas topologias multipulsos e classificando as inúmeras já conhecidas. Estas topologias podem ser classificadas como isoladas ou não-isoladas e também com relação ao número de pulsos na tensão retificada ou na corrente de entrada $(6,12,18,24$ pulsos....), como é mostrado por Singh et alli (2008).

Para transformadores isolados, o defasamento angular requerido pode ser obtido combinando as conexões Estrela, Ziguezague, Delta, Polígono, Scott ou T, conforme Paice (1996) e Schaefer (1965). Para um conversor de 12 pulsos uma forma de se obter sistemas defasados de $30^{\circ}$ entre si é usando as conexões Delta/ Delta-Estrela. O conversor apresenta um primário conectado em Delta e dois secundários, sendo um em Delta em fase com o primário e o outro em Estrela com a defasagem natural de $30^{\circ} \mathrm{com}$ relação ao primário. Outra forma de provocar o ângulo de defasagem correto para um conversor de 12 pulsos é obter um sistema trifásico atrasado de $15^{\circ}$, no secundário do transformador, e outro adiantado de $15^{\circ}$, considerando a rede como referência. Isto pode ser feito utilizando as conexões Delta/Ziguezague-Ziguezague ou Delta/Polígono-Polígono.

Para se obter um conversor de 18 pulsos são necessárias três pontes retificadoras. Neste caso o espectro harmônico das correntes de linha é formado somente pelas ordens harmônicas $\mathrm{k} \cdot 18 \pm 1(\mathrm{k}=1,2,3 \ldots)$. O defasamento angular requerido para o conversor de 18 pulsos é de $20^{\circ}$ entre as pontes retificadoras e pode ser obtido através de inúmeras combinações entre as conexões de transformador, como apresentam Singh et alli (2008) e Paice (1996). Uma forma de se obter um conversor de 18 pulsos isolado é através da conexão Delta/Ziguezague-Estrela-Ziguezague. Nesta configuração, a conexão Estrela presente no secundário apresenta um sistema trifásico defasado de $30^{\circ}$ com relação ao primário que está conectado em Delta, um segundo sistema trifásico secundário é conectado em Ziguezague, este sistema possui um avanço de $20^{\circ}$ com relação ao secundário em Estrela (avanço de $50^{\circ}$ com relação ao primário) e o terceiro sistema trifásico do secundário, também conectado em Ziguezague, possui um atraso de $20^{\circ}$ com relação ao secundário em Estrela (resultando em um atraso de $10^{\circ} \mathrm{com}$ relação ao primário).

Outra maneira de se obter um conversor isolado é promover o isolamento em alta freqüência, introduzindo um estágio CC-CC após o estágio retificador, como é apresentado Seixas e Gonçalves (2007), Seixas e Barbi (2004 e 2001a), garantindo assim, a isolação galvânica. Esta técnica permite que a tensão de saída seja regulada usando uma estratégia de controle apropriada para o estágio intermediário CC-CC.

Este trabalho prioriza os conversores com conexões não-isoladas de transformadores, que empregam conexões especiais de autotransformador (as topologias de autotransformador referidas são as conexões Estrela-diferencial e Delta-diferencial). Um grande número de conexões de autotransformadores pode ser 
encontrado na literatura, como apresentam Singh et alli (2008), Gonçalves (2001) e Burgos et alli (2005).. Uma grande vantagem dessas conexões está no fato de que, somente uma fração da potência total requerida pela carga é processada pelo núcleo magnético do autotransformador (baixa taxa kVA, ou seja, baixa relação entre a potência processada pelo núcleo magnético e potência entregue à carga), resultando em reduzidos pesos e volumes. Por exemplo, o núcleo magnético de um conversor de 12 ou 18 pulsos com conexão Delta-diferencial de autotransformador pode chegar a processar $20 \%$ ou menos da potência requerida pela carga. No entanto, elementos magnéticos adicionais como transformadores de interfase, (IPTs Interphase Transformers), também chamados de reatores ou indutores de interfase são necessários quando as pontes retificadoras de seis pulsos são conectadas em paralelo, isto se deve às diferenças instantâneas entre as tensões das pontes retificadoras. Estas diferenças são responsáveis pelo desbalanceamento das correntes dos secundários do transformador. Além disso, nestas condições não ocorre o cancelamento natural das componentes harmônicas esperadas em conversores multipulsos. Para conversores de 12 pulsos não-isolados, com conexões Estrela ou Delta-diferencial, são necessários quatro IPTs conectados do lado CC. Nas topologias de 18 pulsos são necessários seis IPTs. A fim de melhorar a utilização desses elementos e minimizar o volume adicional introduzido por eles, alguns aprimoramentos têm sido propostos nos últimos anos. Como, por exemplo, o emprego de IPTs ativos que combinam algumas funções dos bloqueadores de seqüência zero (ZSBT - Zero Sequence Blocking Transfromers) e IPTs convencionais. Além disso, esforços para simplificar as estruturas dos autotransformadores e reduzir o número adicional de enrolamentos vêm sendo relatados.

A proposta deste trabalho é apresentar um estudo matemático com relação aos conversores multipulsos CA-CC trifásicos, baseados nas conexões Delta-diferencial ou Estrela-diferencial de autotransformadores, e obter expressões generalizadas que permitam um cálculo rápido e fácil de parâmetros importantes para o projeto de conversores de 12 e 18 pulsos. Através de diagramas fasoriais, expressões trigonométricas são obtidas e generalizadas para qualquer valor de tensões de entrada e saída. Estas expressões apresentam o cálculo para as tensões sobre todos os enrolamentos do autotransformador. Essa análise matemática reúne todas as topologias Delta-diferencial e Estrela-diferencial, elevadoras e abaixadoras de tensão, tanto para conversores de 12 como de 18 pulsos.

Através das expressões gerais obtidas podem-se traçar curvas, que possibilitam a determinação da relação de espiras para todos os enrolamentos secundários do autotransformador, além de suas respectivas polaridades. No entanto, a maior contribuição deste trabalho é a possibilidade de escolher qualquer tensão de saída para o conversor em função de qualquer tensão de entrada e vice-versa.

\section{CONEXÕES ESTRELA-DIFERENCIAIS}

\subsection{Análises das tensões sobre os enrolamentos}

A Figura 1 (a) apresenta o diagrama fasorial para o sistema primário de tensões $\left(V_{a}, V_{b}\right.$ e $\left.V_{c}\right)$ e os sistemas de tensões secundários adequadamente defasados, chamados de $\left(V_{R 1}\right.$, $V_{S 1}$ e $\left.V_{T 1}\right)$ para o sistema em avanço e de $\left(V_{R 2}, V_{S 2}\right.$ e $\left.V_{T 2}\right)$ para o sistema em atraso. $\mathrm{O}$ ângulo $\theta$ determina a operação do conversor em 12 pulsos $\left(\theta=15^{\circ}\right)$ ou 18 pulsos $(\theta=$ $20^{\circ}$ ). O conversor de 18 pulsos requer também um sistema trifásico de tensões em fase com as tensões primárias. Este sistema é denotado por $\left(V_{R n}, V_{S n}\right.$ e $\left.V_{T n}\right)$, como é apresentado por Gonçalves e Seixas (2006a e 2006b), Gonçalves (2001) e por Seixas (2001b).

A Figura 1 (b) representa o núcleo magnético trifásico, com o primário conectado em Estrela, os enrolamentos denominados $N_{a}, N_{b}$ e $N_{c}$ são montados nas pernas (A), (B) e (C) respectivamente. Os secundários são compostos por 15 enrolamentos para o conversor de 18 pulsos e 12 enrolamentos para o conversor de 12 pulsos. Os enrolamentos $N_{a 1}, N_{a 2}, N_{a 3}, N_{a 4}$ e $N_{a n}$ são montados sobre a perna (A), já os enrolamentos $N_{b 1}, N_{b 2}, N_{b 3}, N_{b 4}, N_{b n}$ e $N_{c 1}, N_{c 2}, N_{c 3}, N_{c 4}, N_{c n}$ são posicionados sobre as pernas (B) e (C) respectivamente. A forma como são feitas as conexões destes enrolamentos esta representadas na figura 2. Esta conexão é chamada de genérica, pois, a partir dela podem-se gerar conexões elevadoras ou abaixadoras de tensão para conversores de 12 e 18 pulsos, para a topologia Estrela-diferencial. No caso do conversor de 12 pulsos, os enrolamentos $N_{a n}, N_{b n}$, e $N_{c n}$ apresentados na figura 2 não são necessários. As tensões sobre os enrolamentos são representadas pelos mesmos índices, por exemplo, $V_{a 1}$ corresponde à tensão sobre o enrolamento $N_{a 1}$. É importante mencionar que o índice $\mathrm{n}$ (por exemplo, $N_{a n}$ ) refere-se ao ponto neutro e não denota o enésimo enrolamento.

Os sistemas de tensão apropriadamente defasados são obtidos a partir de enrolamentos secundários auxiliares. Logo, as tensões resultantes de saída são formadas pela composição das tensões de entrada e das tensões nos enrolamentos secundários auxiliares.

As tensões são consideradas positivas quando estão de acordo com a direção (polaridade) das tensões de referência (sistema de tensão primário). 


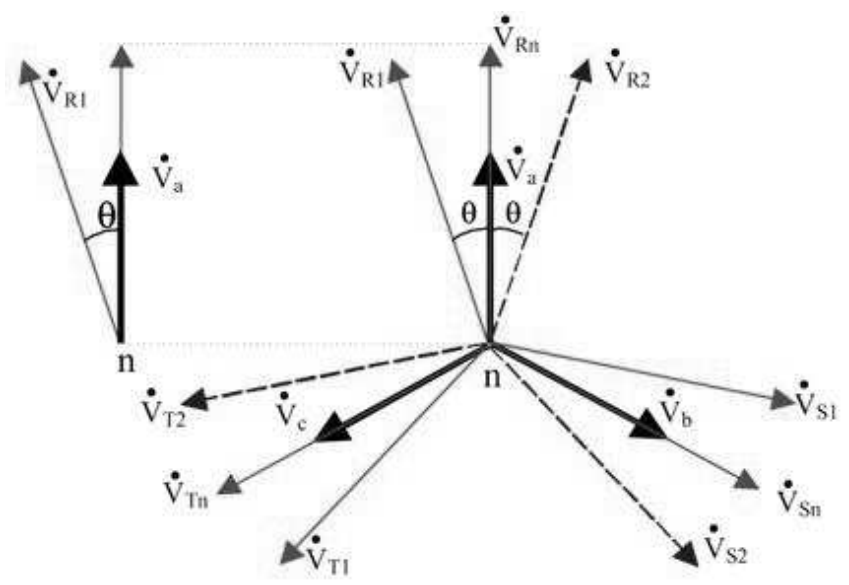

(a)

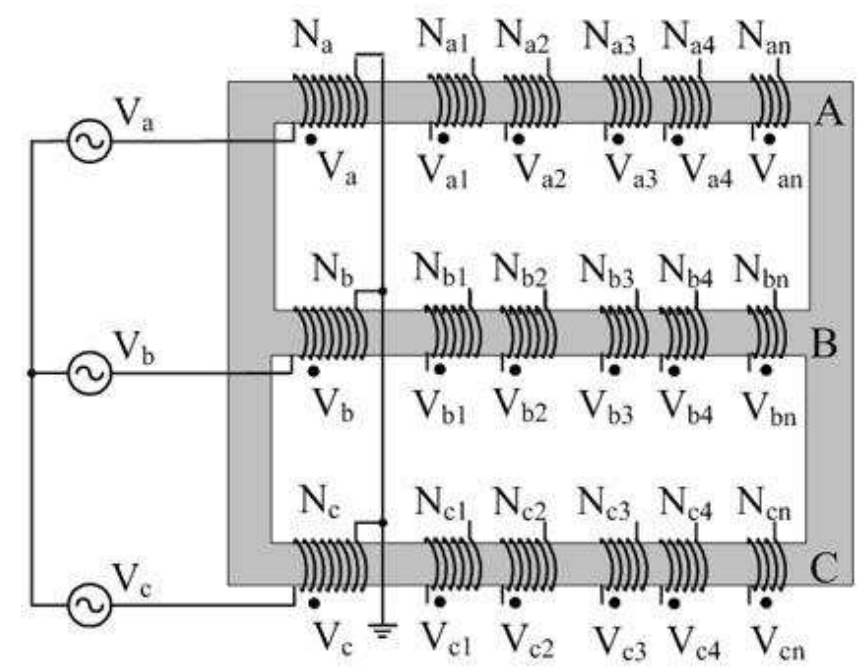

(b)

Figura 1: Diagrama fasorial para o autotransformador com conexão Estrela-diferencial (a) e esquema das bobinas montadas sobre o núcleo magnético (b).

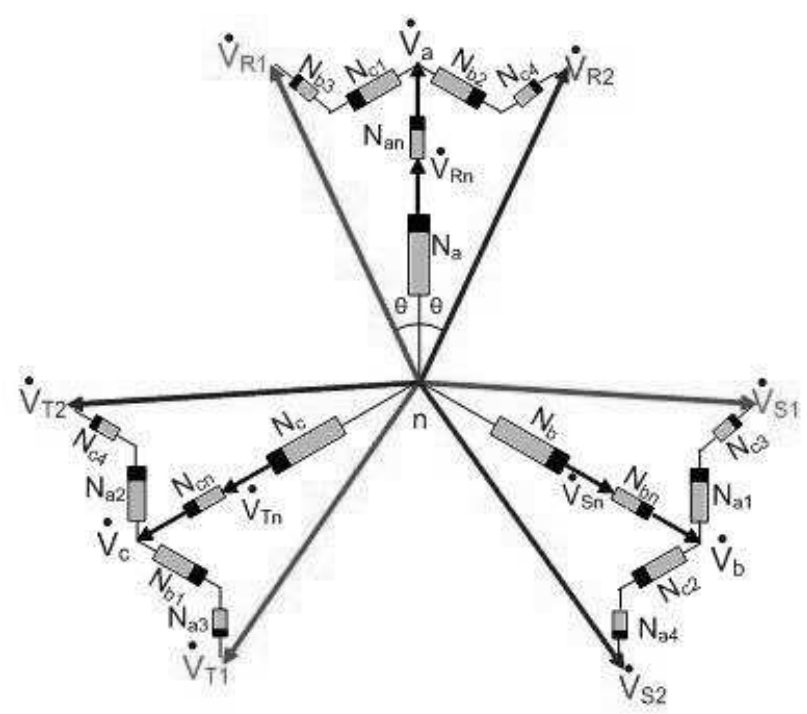

Figura 2: Polaridades e conexões dos enrolamentos para o autotransformador.

\subsubsection{Equações para $V_{c 1}>0$ e $V_{b 3}>0$ :}

A Figura 3 (a) apresenta o diagrama fasorial simplificado quando as tensões são positivas nos enrolamentos $N_{c 1}$ e $N_{b 3}$. A tensão auxiliar $V_{x}$ é definida entre o ponto neutro e o ponto comum dos enrolamentos $N_{c 1}$ e $N_{b 3}$. O ângulo chamado de é definido como o ângulo entre as tensões $V_{x}$ e $V_{a}$ (tensão de referência). As tensões $V_{c 1}$ e $V_{b 3}$ têm a mesma direção de $V_{c}$ e $V_{b}$, respectivamente.

Considerando os triângulos formados pelo módulo das tensões $\left(V_{x}, V_{R 1}\right.$ e $\left.V_{b 3}\right)$ e $\left(V_{x}, V_{c 1}\right.$ e $\left.V_{a}\right)$, apresentados na figura 3 (a), as seguintes expressões podem ser escritas:

$$
\frac{V_{x}}{\operatorname{sen}\left(120^{\circ}+\theta\right)}=\frac{V_{R 1}}{\operatorname{sen}\left(60^{\circ}-\alpha\right)}=\frac{V_{b 3}}{\operatorname{sen}(\alpha-\theta)}
$$

$$
\frac{V_{x}}{\operatorname{sen}\left(60^{\circ}\right)}=\frac{V_{a}}{\operatorname{sen}\left(120^{\circ}-\alpha\right)}=\frac{V_{c 1}}{\operatorname{sen}(\alpha)}
$$

Para esta condição (tensões $V_{c 1}$ e $V_{b 3}$ são valores positivos), $\alpha$ varia entre $\theta$ e $60^{\circ}$ em relação a referência (tensão primária). Logo,

Para $\alpha=60^{\circ}$ e $\theta=0^{\circ} \rightarrow V_{c 1}=V_{x}=V_{b 3}=V_{a} \rightarrow V_{R 1}=0$;

Para $\alpha=\theta \rightarrow V_{b 3}=0$.

A tensão resultante no secundário $\left(V_{R 1}\right)$ é sempre menor que a tensão de entrada $\left(V_{a}\right)$. Essa é uma situação particular na qual se tem uma conexão abaixadora (step-down). 

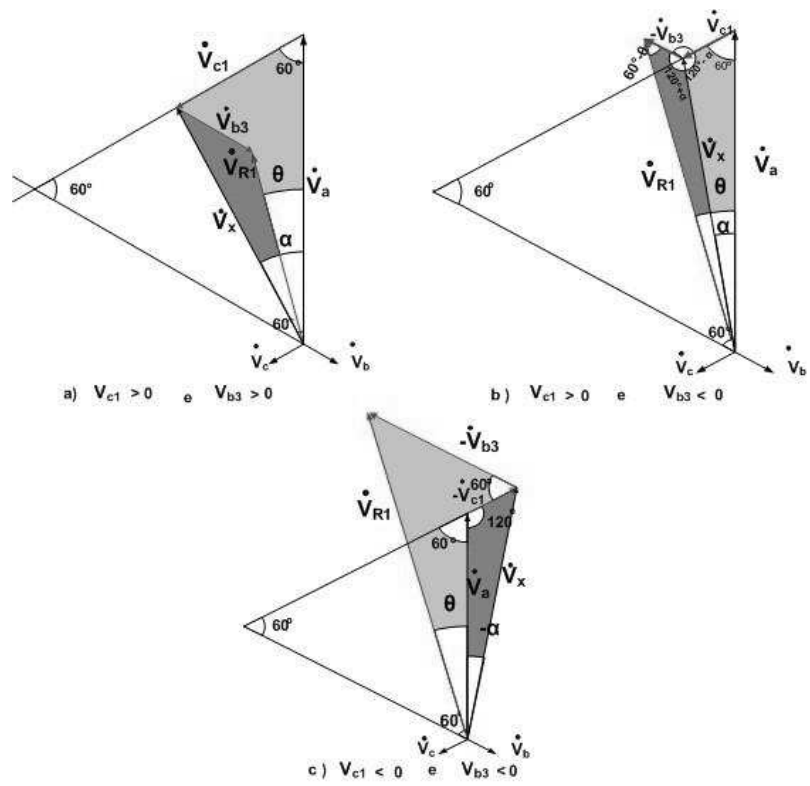

Figura 3: Diagramas fasoriais para as topologias Estrela-diferencial.

\subsubsection{Equações para $V_{c 1}>0$ e $V_{b 3}<0$ :}

Quando $N_{b 3}$ tem polaridade invertida, resulta em uma tensão $V_{b 3}$ negativa, $\alpha$ está entre $\theta$ e $0^{\circ}$. A tensão de saída $\left(V_{R 1}\right)$ pode ser menor, igual ou maior que a tensão de entrada. A Figura 3 (b) apresenta o diagrama fasorial simplificado para esta condição.

A partir dos triângulos em destaque na figura 3 (b), obtêm-se as seguintes expressões:

$$
\begin{gathered}
\frac{V_{x}}{\operatorname{sen}\left(60^{\circ}\right)}=\frac{V_{a}}{\operatorname{sen}\left(120^{\circ}-\alpha\right)}=\frac{V_{c 1}}{\operatorname{sen}(\alpha)} \\
\frac{V_{x}}{\operatorname{sen}\left(60^{\circ}-\theta\right)}=\frac{V_{R 1}}{\operatorname{sen}\left(120^{\circ}+\alpha\right)}=\frac{-V_{b 3}}{\operatorname{sen}(\theta-\alpha)}
\end{gathered}
$$

\subsubsection{Equações para $V_{c 1}<0$ e $V_{b 3}<0$ :}

Neste caso, $V_{c 1}$ e $V_{b 3}$ assumem valores negativos e varia entre $0^{\circ}$ e $-60^{\circ}$. A tensão $V_{R 1}$ é maior que a tensão de entrada, ou seja, tem-se a conexão elevadora de tensão (step-up). A Figura 3 (c) mostra o diagrama fasorial para esta condição e as equações associadas a este diagrama são:

$$
\frac{V_{x}}{\operatorname{sen}\left(120^{\circ}\right)}=\frac{V_{a}}{\operatorname{sen}\left(60^{\circ}+\alpha\right)}=\frac{-V_{c 1}}{\operatorname{sen}(-\alpha)}
$$

$$
\frac{V_{x}}{\operatorname{sen}\left(60^{\circ}-\theta\right)}=\frac{V_{R 1}}{\operatorname{sen}\left(120^{\circ}+\alpha\right)}=\frac{-V_{b 3}}{\operatorname{sen}(\theta-\alpha)}
$$

\subsection{Expressão genérica}

Considerando as expressões de (1) a (6) e usando identidades trigonométricas é possível obter uma única expressão que descreve as tensões através dos enrolamentos secundários em função da tensão de referência $\left(V_{a}\right)$ e dos ângulos $\alpha$ e $\theta$. Esta expressão é apresentada em (7). As tensões $V_{R 1}, V_{c 1}$ e $V_{b 3}$ são facilmente determinadas em (8), (9) e (10) respectivamente.

$$
\begin{aligned}
V_{a} \frac{\operatorname{sen}\left(60^{\circ}\right)}{\operatorname{sen}\left(120^{\circ}-\alpha\right)}=V_{c 1} \frac{\operatorname{sen}\left(60^{\circ}\right)}{\operatorname{sen}(\alpha)} & = \\
=V_{R 1} \frac{\operatorname{sen}\left(120^{\circ}+\theta\right)}{\operatorname{sen}\left(60^{\circ}-\alpha\right)} & =V_{b 3} \frac{\operatorname{sen}\left(120^{\circ}+\theta\right)}{\operatorname{sen}(\alpha-\theta)}
\end{aligned}
$$

Através da equação (8) para uma tensão de fase $\left(V_{a}\right)$ conhecida, escolhe-se um valor para a tensão de fase resultante nos secundários $V_{R 1}$ e calcula-se o valor do ângulo auxiliar $\alpha$. Com os valores de $\alpha$ e $V_{a}$, se obtém os valores para $V_{c 1}$ e $V_{b 3}$ através das expressões (9) e (10).

$$
\begin{gathered}
V_{R 1}=V_{a} \cdot \frac{\operatorname{sen}\left(60^{\circ}\right)}{\operatorname{sen}\left(120^{\circ}-\alpha\right)} \cdot \frac{\operatorname{sen}\left(60^{\circ}-\alpha\right)}{\operatorname{sen}\left(120^{\circ}+\theta\right)} \\
V_{c 1}=V_{a} \cdot \frac{\operatorname{sen}\left(60^{\circ}\right)}{\operatorname{sen}\left(120^{\circ}-\alpha\right)} \cdot \frac{\operatorname{sen}(\alpha)}{\operatorname{sen}\left(60^{\circ}\right)} \\
V_{b 3}=V_{a} \cdot \frac{\operatorname{sen}\left(60^{\circ}\right)}{\operatorname{sen}\left(120^{\circ}-\alpha\right)} \cdot \frac{\operatorname{sen}(\alpha-\theta)}{\operatorname{sen}\left(120^{\circ}+\theta\right)}
\end{gathered}
$$

\subsection{Enrolamentos adicionais conversores de 18 pulsos}

Os conversores de 18 pulsos necessitam de um terceiro sistema de tensões secundárias, o qual está em fase com as tensões primárias e as tensões da rede. As tensões do sistema adicional $V_{R n}, V_{S n}$ e $V_{T n}$ devem ter o mesmo valor em módulo das tensões dos sistemas defasados de $+20^{\circ}\left(V_{R 1}, V_{S 1}, V_{T 1}\right) \mathrm{e}$ $-20^{\circ}\left(V_{R 2}, V_{S 2}, V_{T 2}\right)$, como mostra a figura 1 (a). Assim, o enrolamento $N_{a n}$ é montado na mesma perna do enrolamento $N_{a}$, e gera a tensão $V_{a n}$ que está em fase com a tensão $V_{a}$, o mesmo é válido para os enrolamentos $N_{b n}$ e $N_{c n}$. A amplitude da tensão através de $N_{a n}$ é apresentada na equação (11).

$$
V_{a n}=V_{R 1}-V_{a}
$$


É importante relembrar que o conversor de 12 pulsos não requer esses enrolamentos adicionais, pois possui apenas dois sistemas defasados, um adiantado de $+15^{\circ}$ e outro atrasado de $-15^{\circ}$.

\subsection{Relações de espiras}

As relações de espiras entre os enrolamentos do autotransformador são obtidas através das relações entre as tensões aplicadas sobre os enrolamentos secundários e primários. O valor de $K_{a}$ é definido como o quociente entre $V_{a n}$ e $V_{a}$ como se pode ver na equação (12).

$$
K_{a}=\frac{N_{a n}}{N_{a}}=\frac{V_{a n}}{V_{a}}
$$

A relação entre $V_{c 1}$ e $V_{a}$ define o valor de $K_{b}$, como é mostrado em (13).

$$
K_{b}=\frac{N_{c 1}}{N_{a}}=\frac{V_{c 1}}{V_{a}}
$$

Finalmente a equação (14) apresenta a relação entre $V_{b 3}$ e $V_{a}$ e define o valor de $K_{c}$.

$$
K_{c}=\frac{N_{b 3}}{N_{a}}=\frac{V_{b 3}}{V_{a}}
$$

As polaridades dos enrolamentos são adotadas positivas quando eles estão na mesma direção de seus respectivos enrolamentos primários. Assim, se uma tensão secundária resultar em um valor negativo, seu enrolamento tem polaridade oposta ao enrolamento primário montado na mesma perna do núcleo. Como consequiência o valor da relação de espiras é negativo.

A partir da expressão (8) chega-se a expressão (15), que apresenta o valor do ângulo auxiliar $\alpha$.

$$
\alpha=\operatorname{arctg}\left[\frac{3 \cdot V_{a}}{2 \cdot V_{R 1} \cdot(\sqrt{3} \cdot \cos (\theta)-\operatorname{sen}(\theta))-\sqrt{3} \cdot V_{a}}\right]
$$

\subsection{Curvas para os conversores de 12 e 18 pulsos com conexão Estrela-diferencial}

As Figuras 4 e 5 apresentam ábacos que permitem obter os valores das relações de espiras, sem a necessidade dos cálculos apresentados anteriormente. Observa-se pelas curvas que os valores das relações de espiras para uma mesma tensão de saída, não são os mesmos, se a tensão de entrada for diferente, ou seja, para cada tensão de entrada tem-se uma curva distinta. Escolhendo-se a tensão de saída desejada $\left(V_{R 1}\right)$ e sabendo-se qual é a tensão de entrada, é simples e rápido obter as relações entre as tensões sobre os enrolamentos e obter os valores de $K_{a}, K_{b}$ e $K_{c}$, a partir das curvas apresentadas para os conversores de 12 e 18 pulsos.

A Figura 4 apresenta curvas para os conversores de 12 pulsos $\left(\theta=15^{\circ}\right)$, este conversor necessita apenas de dois enrolamentos secundários por fase. Logo, tem-se apenas as relações $K_{b}$ na figura 4(a) e $K_{c}$ na figura 4(b).

A Figura 5 apresenta curvas para os conversores de 18 pulsos $\left(\theta=20^{\circ}\right)$, este conversor necessita de um enrolamento a mais por fase, logo se tem as relações $K_{a}$ na figura 5 (a), $K_{b}$ na figura 5 (b) e $K_{c}$ na figura 5 (c).

Quando uma relação de espiras é nula, significa que o enrolamento não existe e recai em um caso especial de conexão. Por exemplo, quando $K_{c}$ é nulo, $V_{b 3}=0$ e se tem $\alpha=\theta$.

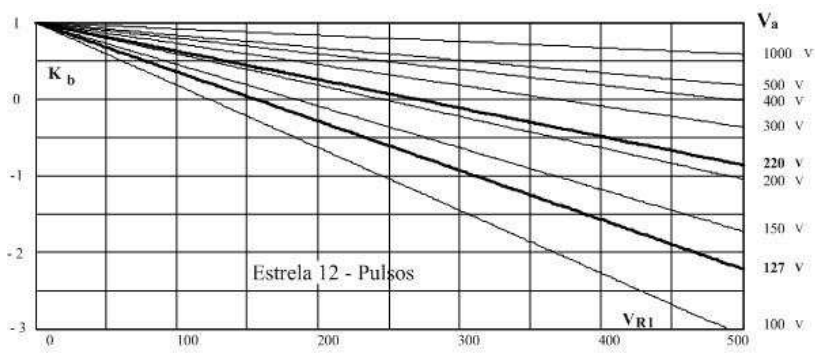

(a)

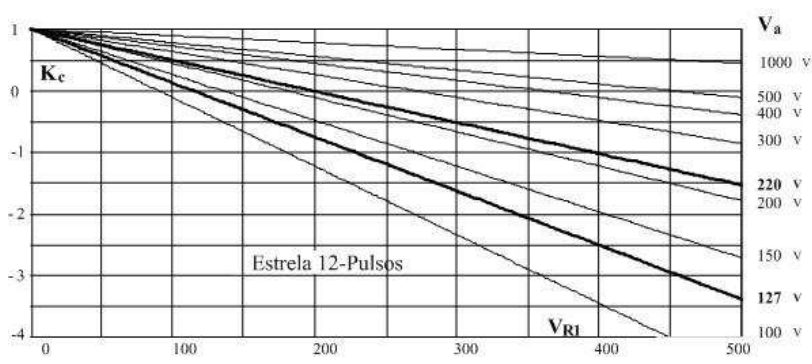

(b)

Figura 4: Conversor de 12 pulsos com conexão Estrela-diferencial. Curvas para $K_{b}(\mathrm{a})$ e $K_{c}$ (b) 


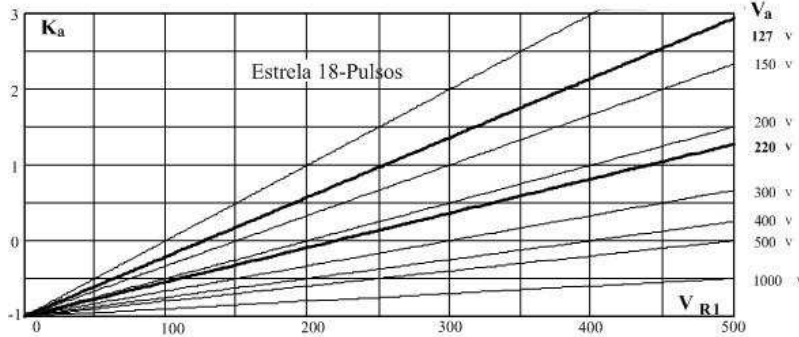

(a)

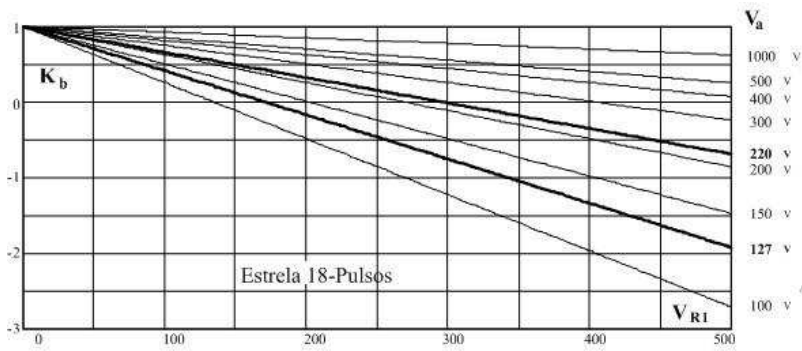

(b)

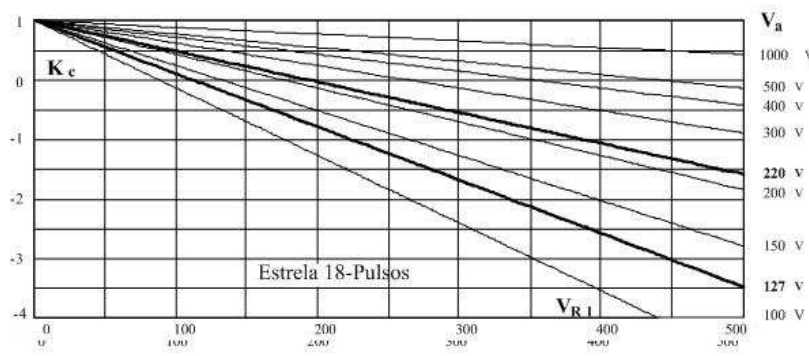

(c)

Figura 5: Conversor de 18 pulsos com conexão Estrela-diferencial. Curvas para $K_{a}$ (a), $K_{b}$ (b) e $K_{c}$ (c).

\section{CONEXÕES DELTA-DIFERENCIAIS}

\subsection{Análises das tensões sobre os enrolamentos}

A análise das tensões para a conexão Delta-diferencial é similar à apresentada para a conexão Estrela-diferencial como apresenta Gonçalves e Seixas (2006b). No entanto, as tensões aplicadas sobre os enrolamentos primários são tensões de linha $\left(V_{a b}, V_{b c}\right.$ e $\left.V_{c a}\right)$. A Figura 6 (a) apresenta o diagrama fasorial para a topologia Delta. O diagrama fasorial apresenta o sistema trifásico de tensões primárias $\left(V_{a b}, V_{b c} \mathrm{e}\right.$ $\left.V_{c a}\right)$, as tensões de fase e de linha do sistema adiantado de $\theta^{\circ}$ $\left(V_{R 1}, V_{S 1}\right.$ e $\left.V_{T 1}\right)$ e $\left(V_{R S 1}, V_{S T 1}\right.$ e $\left.V_{T R 1}\right)$, as tensões de fase e de linha do sistema atrasado de $\theta^{\circ}\left(V_{R 2}, V_{S 2}\right.$ e $\left.V_{T 2}\right)$ e $\left(V_{R S 2}, V_{S T 2}\right.$ e $\left.V_{T R 2}\right)$, e o sistema em fase com as tensões primárias $\left(V_{R n}\right.$, $V_{S n}$ e $\left.V_{T n}\right)$.
Para o conversor de 18 pulsos o terceiro sistema trifásico em fase com a rede é necessário, as tensões devem ter o mesmo valor em módulo das demais tensões secundárias resultantes. No caso do conversor Delta-diferencial de 18 pulsos são necessários dois enrolamentos auxiliares a mais por fase em comparação com a topologia Estrela, que utiliza apenas um enrolamento auxiliar por fase para gerar o terceiro sistema trifásico. A Figura 6 (b) apresenta o núcleo magnético para a conexão Delta-diferencial. Assim, a conexão Delta de 12 pulsos $\left(\theta=15^{\circ}\right)$ é composta pelos enrolamentos primários e 12 enrolamentos secundários auxiliares, já a conexão de 18 pulsos $\left(\theta=20^{\circ}\right)$ necessita de 18 enrolamentos secundários.

Os enrolamentos primários são definidos como $N_{a b}, N_{b c}$ e $N_{c a}$. Os enrolamentos secundários $N_{a b 1}, N_{a b 2}, N_{a b 3}, N_{a b 4}$, $N_{a b n}$ e $N_{a b n}$ são montados sobre uma mesma perna (A) do núcleo, junto ao enrolamento $N_{a b}$. Os enrolamentos $N_{b c 1}, N_{b c 2}, N_{b c 3}, N_{b c 4}, N_{b c n}$ e $N b c n 1$ são montados em uma mesma perna do núcleo (B), juntamente com o enrolamento primário $N_{b c}$. Finalmente, os enrolamentos $N_{c a 1}, N_{c a 2}$, $N_{c a 3}, N_{c a 4}, N_{c a n}$ e $N_{c a n 1}$ são montados na perna (C) do núcleo, juntamente com o enrolamento primário $N_{c a}$. Estes enrolamentos são conectados de acordo com a Figura 7, que apresenta todos os enrolamentos presentes na conexão Delta-diferencial generalizada. Para o conversor de 12 pulsos os enrolamentos $N_{a b n}, N_{a b n 1}, N_{b c n}, N_{b c n 1}, N_{c a n}$, e $N_{c a n 1}$ não são necessários.

\subsubsection{Equações para $V_{c a 1}>0$ e $V_{b c 3}>0$ :}

A Figura 8 apresenta diagramas fasoriais para as tensões através dos enrolamentos $N_{c a 1}$ e $N_{b c 3}$. Como foi apresentado para a topologia Estrela, a tensão auxiliar $V_{x}$ é definida como a tensão entre o neutro e o ponto comum entre as tensões secundárias sobre os enrolamentos $N_{c a 1}$ e $N_{b c 3}$. Considerando os triângulos $\left(V_{x}, V_{c a 1}\right.$ e $\left.V_{a}\right)$ e $\left(V_{x}, V_{R 1}\right.$ e $\left.V_{b c 3}\right)$, apresentado na figura 8 (a), são válidas as expressões (16) e (17).

$$
\begin{gathered}
\frac{V_{x}}{\operatorname{sen}\left(30^{\circ}\right)}=\frac{V_{a}}{\operatorname{sen}\left(150^{\circ}-\alpha\right)}=\frac{V_{c a 1}}{\operatorname{sen}(\alpha)} \\
\frac{V_{x}}{\operatorname{sen}\left(90^{\circ}+\theta\right)}=\frac{V_{R 1}}{\operatorname{sen}\left(90^{\circ}-\alpha\right)}=\frac{V_{b c 3}}{\operatorname{sen}(\alpha-\theta)}
\end{gathered}
$$

A partir da figura 8 (a) observa-se que $\alpha$ varia de $\theta$ a $90^{\circ}$. Assim, os limites encontrados são:

Para $\alpha=90^{\circ} \rightarrow V_{c a 1}=2, V_{b c 3}=V_{a} \cdot \cos \left(30^{\circ}\right)$ e $V_{R 1}=0 ;$

Para $\alpha=\theta \rightarrow V_{c 3}=0$.

A tensão de saída no secundário do autotransformador $\left(V_{R 1}\right)$ é sempre menor que a tensão de fase de entrada $\left(V_{a}\right)$. Por este 


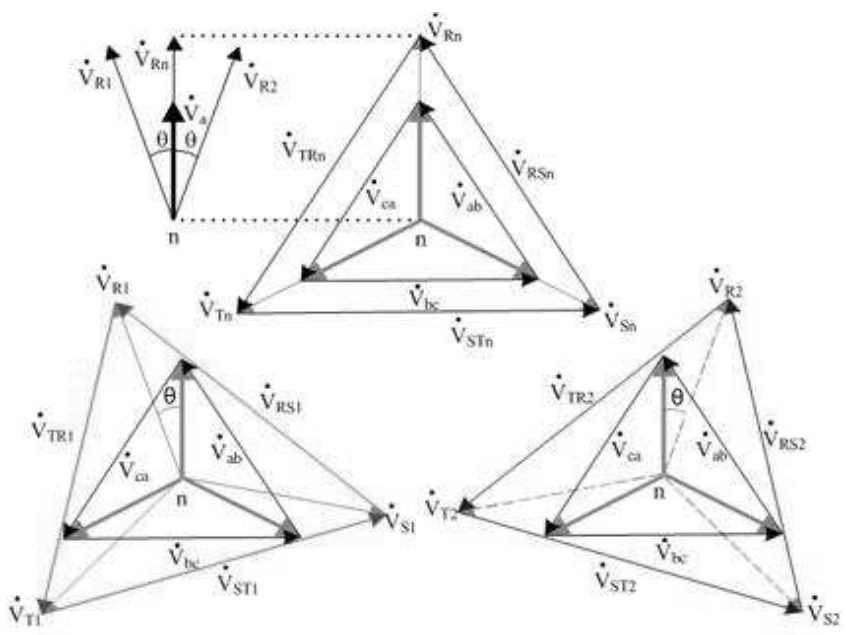

(a)

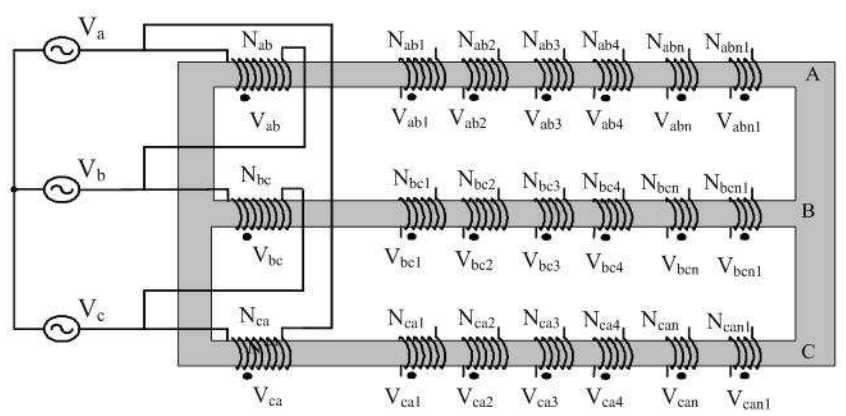

(b)

Figura 6: Diagrama fasorial para a topologia Delta-diferencial (a) e esquema das bobinas montadas sobre o núcleo magnético (b).

motivo, esta conexão é chamada abaixadora de tensão.

\subsubsection{Equações para $V_{c a 1}>0$ e $V_{b c 3}<0$ :}

Para este caso o diagrama fasorial é apresentado na Figura 8 (b), a partir dele, obtêm-se as expressões (18) e (19).

$$
\begin{gathered}
\frac{V_{x}}{\operatorname{sen}\left(30^{\circ}\right)}=\frac{V_{a}}{\operatorname{sen}\left(150^{\circ}-\alpha\right)}=\frac{V_{c a 1}}{\operatorname{sen}(\alpha)} \\
\frac{V_{x}}{\operatorname{sen}\left(90^{\circ}-\theta\right)}=\frac{V_{R 1}}{\operatorname{sen}\left(90^{\circ}+\alpha\right)}=\frac{-V_{b c 3}}{\operatorname{sen}(\theta-\alpha)}
\end{gathered}
$$

O ângulo $\alpha$ varia entre $\theta$ e $0^{\circ}$, e $V_{R 1}$ pode ser menor, igual ou maior que a tensão de entrada.

\subsubsection{Equações para $V_{c a 1}<0$ e $V_{b c 3}<0$ :}

Neste caso, $\alpha$ varia entre $0^{\circ} \mathrm{e}-30^{\circ}$, enquanto $V_{R 1}$ é maior que $V_{a}$, como mostra o diagrama fasorial da figura 8(c). As

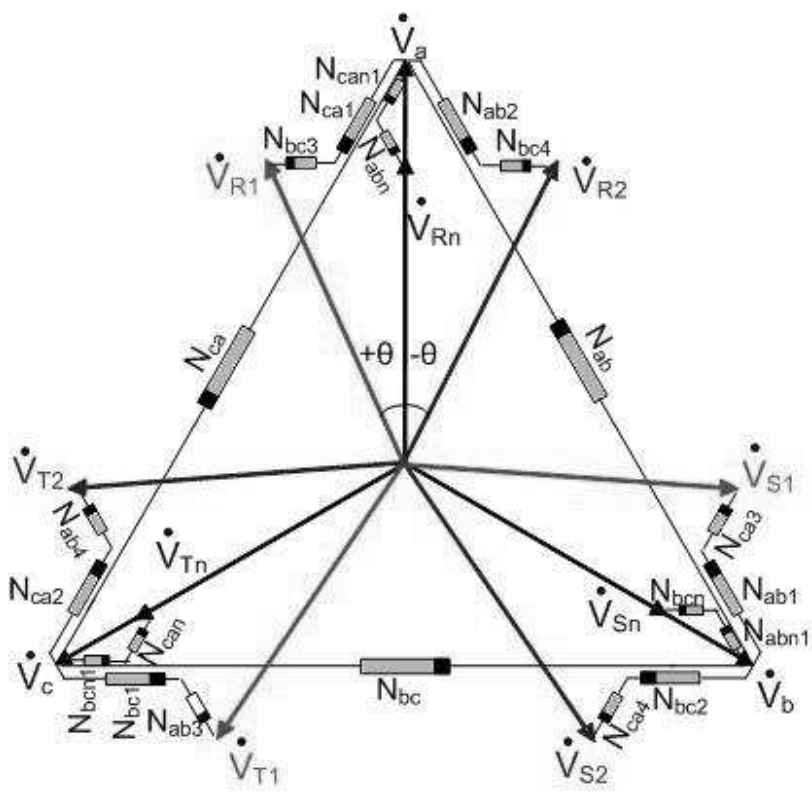

Figura 7: Polaridades e conexões dos enrolamentos para o autotransformador.

expressões (20) e (21) são obtidas a partir deste diagrama.

$$
\begin{gathered}
\frac{V_{x}}{\operatorname{sen}\left(150^{\circ}\right)}=\frac{V_{a}}{\operatorname{sen}\left(30^{\circ}+\alpha\right)}=\frac{-V_{c a 1}}{\operatorname{sen}(-\alpha)} \\
\frac{V_{x}}{\operatorname{sen}\left(90^{\circ}-\theta\right)}=\frac{V_{R 1}}{\operatorname{sen}\left(90^{\circ}+\alpha\right)}=\frac{-V_{b c 3}}{\operatorname{sen}(\theta-\alpha)}
\end{gathered}
$$

\subsection{Expressão genérica}

Usando o mesmo procedimento adotado para a conexão Estrela de autotransformador, uma expressão única é obtida e apresentada em (22). Essa expressão está definida em função dos ângulos $\alpha, \theta$ e da tensão de entrada e descreve as tensões através de todos os enrolamentos secundários.

$$
\begin{aligned}
& V_{a} \frac{\operatorname{sen}\left(30^{\circ}\right)}{\operatorname{sen}\left(150^{\circ}-\alpha\right)}=V_{c a 1} \frac{\operatorname{sen}\left(30^{\circ}\right)}{\operatorname{sen}(\alpha)}= \\
& =V_{R 1} \frac{\operatorname{sen}\left(90^{\circ}-\theta\right)}{\operatorname{sen}\left(90^{\circ}+\alpha\right)}=V_{b c 3} \frac{\operatorname{sen}\left(90^{\circ}+\theta\right)}{\operatorname{sen}(\alpha-\theta)}
\end{aligned}
$$

Os valores para $V_{R 1}, V_{c a 1}$ e $V_{b c 3}$ podem se calculados através das expressões (23) a (25), respectivamente.

$$
V_{R 1}=V_{a} \cdot \frac{\operatorname{sen}\left(30^{\circ}\right)}{\operatorname{sen}\left(150^{\circ}-\alpha\right)} \cdot \frac{\operatorname{sen}\left(90^{\circ}+\alpha\right)}{\operatorname{sen}\left(90^{\circ}-\theta\right)}
$$



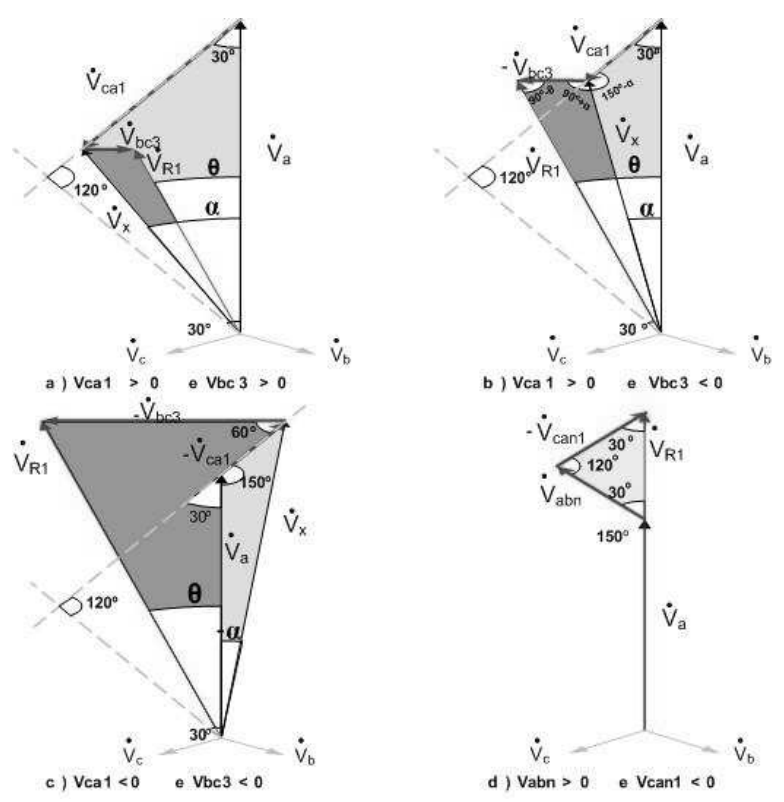

Figura 8: Diagramas fasoriais para as topologias Delta-diferencial.

$$
\begin{gathered}
V_{c a 1}=V_{a} \cdot \frac{\operatorname{sen}\left(30^{\circ}\right)}{\operatorname{sen}\left(150^{\circ}-\alpha\right)} \cdot \frac{\operatorname{sen}(\alpha)}{\operatorname{sen}\left(30^{\circ}\right)} \\
V_{b c 3}=V_{a} \cdot \frac{\operatorname{sen}\left(30^{\circ}\right)}{\operatorname{sen}\left(150^{\circ}-\alpha\right)} \cdot \frac{\operatorname{sen}(\alpha-\theta)}{\operatorname{sen}\left(90^{\circ}+\theta\right)}
\end{gathered}
$$

\subsection{Enrolamentos adicionais conversores de 18 pulsos}

Neste caso, são necessários dois enrolamentos a mais por fase para gerar um terceiro sistema trifásico, em fase com as tensões de fase do primário do autotransformador e com as tensões da rede. Os enrolamentos adicionais $N_{a b n}$ e $N_{a b n 1}$ são montados sobre a mesma perna do núcleo junto ao enrolamento primários $N_{a b}$ as tensões sobre estes enrolamentos secundários são $V_{a b n}$ e $V_{a b n 1}$ em fase com $V_{a}$. A soma de $V_{a b}, V_{a b n}$ e $V_{c a n 1}$ resulta em $V_{R n}$, que deve possuir mesma amplitude de $V_{R 1}$ e $V_{R 2}$ e estar em fase com a referência $\left(V_{a}\right)$, como mostrado na figure 8 (d). A tensão através dos enrolamentos secundários $N_{a b n}$ e $N_{a b n 1}$ é apresentada na equação (26).

$$
V_{a b n}=\frac{V_{R 1}-V_{a}}{2 \cdot \cos \left(30^{\circ}\right)}
$$

\subsection{Relações de espiras}

A relação de espiras $K_{a}$ é apresentada na equação (27). O valor de $K_{a}$ é obtido pela relação entre as tensões $V_{a b n}$ ou
$V_{a b n 1}$ e a tensão primária de linha $V_{a b}$.

$$
K_{a}=\frac{N_{a b n}}{N_{a b}}=\frac{V_{a b n}}{V_{a b}}
$$

A relação entre as tensões $V_{c a 1}$ e $V_{a}$ define $K_{b}$, representado por (28).

$$
K_{b}=\frac{N_{c a 1}}{N_{c a}}=\frac{V_{c a 1}}{V_{c a}}
$$

Finalmente, $K_{c}$ está apresentado na equação (29).

$$
K_{c}=\frac{N_{b c 3}}{N_{b c}}=\frac{V_{b c 3}}{V_{b c}}
$$

Rearranjando a equação (23), é possível obter a equação (30) que apresenta o cálculo do ângulo $\alpha$.

$$
\alpha=-\operatorname{arctg}\left[\frac{1}{3} \cdot \frac{V_{R 1} \cdot \cos (\theta)-V_{a}}{\left(V_{R 1} \cdot \cos (\theta)\right)} \cdot \sqrt{3}\right]
$$

\subsection{Curvas para os conversores de 12 e 18 pulsos com conexão Delta-Diferencial}

Da mesma maneira como foram obtidas as curvas para a conexão Estrela-diferencial, foram obtidas curvas para a conexão Delta-diferencial. Estas curvas estão apresentadas nas Figuras 9 e 10. Elas facilitam encontrar as relações de espiras $K_{a}, K_{b}$ e $K_{c}$ de forma rápida e fácil, basta escolher a curva que corresponde à tensão de entrada e depois entrar com o valor de tensão secundária desejada.

A Figura 9 apresenta curvas para os conversores de 12 pulsos $\left(\theta=15^{\circ}\right)$. Este conversor necessita apenas de dois enrolamentos secundários por fase, logo se têm apenas as relações $K_{b}\left(V_{c a 1} / V_{a}\right)$ figura 9 (a) e $K_{c}\left(V_{b c 3} / V_{a}\right)$ figura 9 (b).

A Figura 10 apresenta curvas para os conversores de 18 pulsos $\left(\theta=20^{\circ}\right), K_{a}$ na figura $10(\mathrm{a}), K_{b}$ na figura 10 (b) e $K_{c}$ na figura $10(\mathrm{c})$.

\section{GENERALIZAÇÃO DAS CONEXÕES ESTRELA E DELTA-DIFERENCIAIS}

Existe uma grande semelhança entre as expressões (7) e (22). Este fato é entendido como uma oportunidade para que estas expressões sejam reescritas, a fim de se obter uma única expressão que represente ambas as topologias Delta e Estrela de autotransformador para conversores de 12 e 18 . 


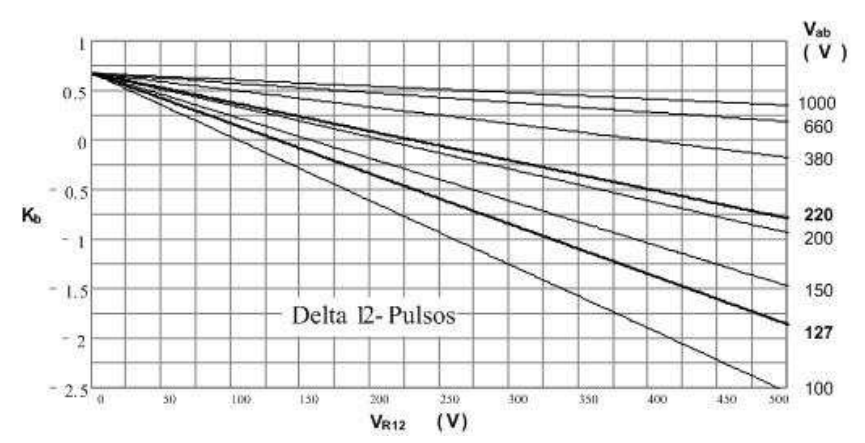

(a)

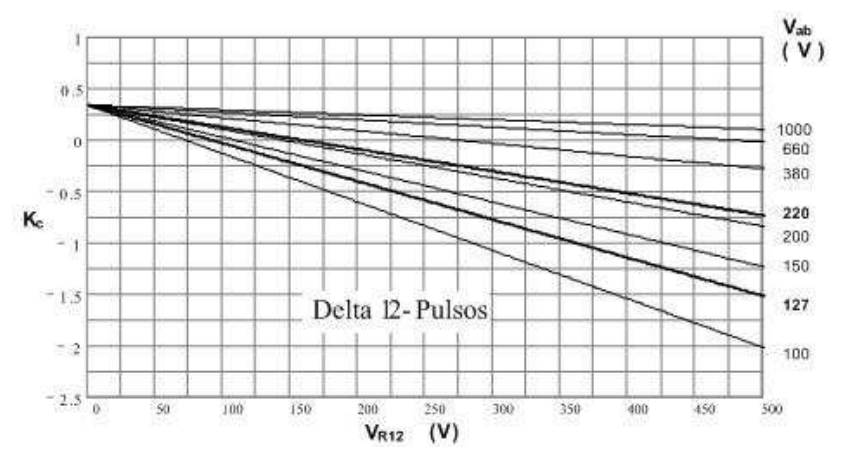

(b)

Figura 9: Conversores de 12 pulsos com conexão Delta-diferencial. Curvas para $K_{b}$ (a) e $K_{c}$ (b).

A principal diferença entre as conexões Estrela-diferencial e Delta-diferencial é que, no caso da conexão Estrela, as tensões secundárias relacionam tensões de fase e, na conexão Delta, relacionam tensões de linha. É importante esclarecer que a conexão que será apresentada não é uma nova conexão. Será apresentado um novo equacionamento que generaliza as conexões Estrela e Delta apresentadas anteriormente. Com este equacionamento não serão necessárias mais equações distintas para as topologias Estrela e Delta de transformador, elas serão representadas por uma única expressão que representará as duas famílias de autotransformadores para conversores de 12 ou 18 pulsos.

\subsection{Diagrama fasorial generalizado}

O ângulo entre as tensões de fase da conexão Estrela e a tensão de linha da conexão Delta é de $30^{\circ}$. Assim para a conexão Delta o ângulo entre $V_{a}$ e $V_{c a 1}$ é $30^{\circ}$, enquanto que, para a conexão Estrela o ângulo entre $V_{a}$ e $V_{c 1}$ é de $60^{\circ}$. Considerando um ângulo adicional $\Psi$, o diagrama fasorial apresentado na figura 8 pode ser reescrito considerando agora o ângulo $\Psi$ como mostra a figura 11. Este diagrama representa ambas as conexões Estrela-diferencial (quando $\Psi$ $=30^{\circ}$ ) e Delta-diferencial (quando $\Psi=0^{\circ}$ ).

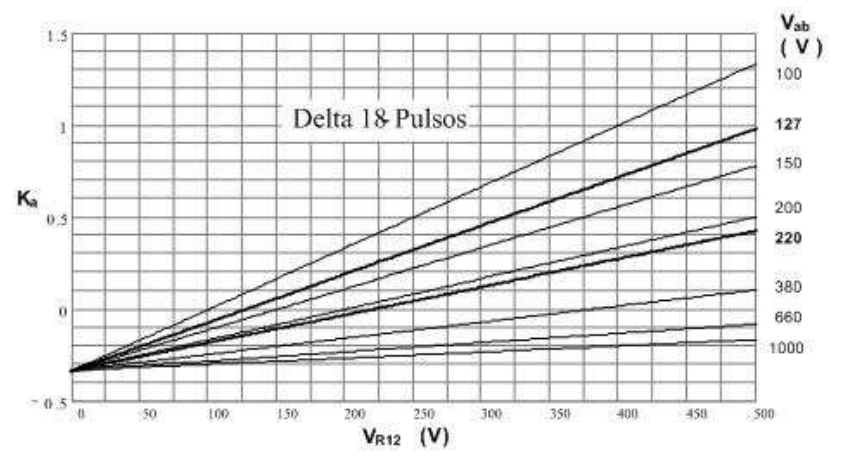

(a)

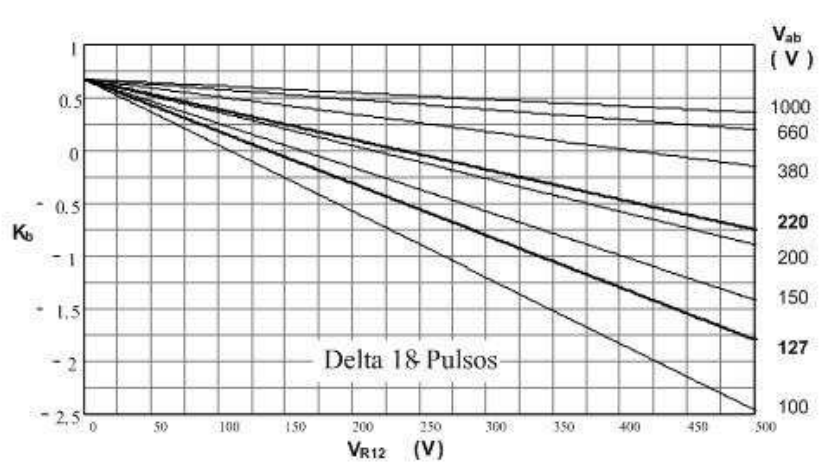

(b)

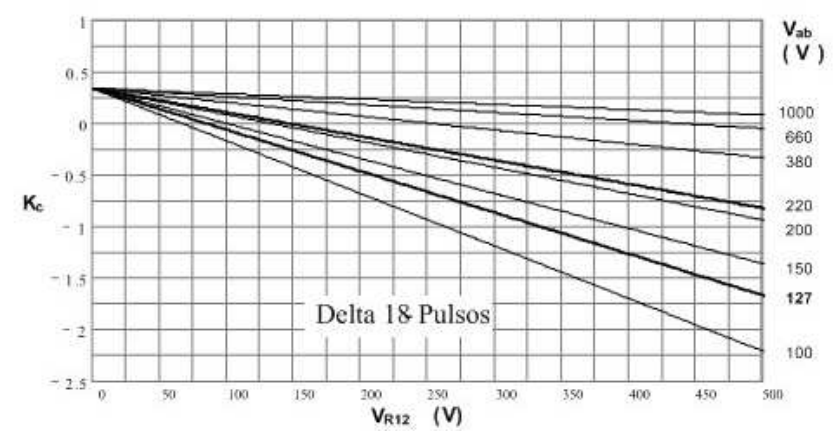

(c)

Figura 10: Conversores de 18 pulsos com conexão Delta-diferencial. Curvas para $K_{a}(\mathrm{a}), K_{b}$ (b) e $K_{c}$ (c).

O diagrama fasorial da figura 11 é utilizado para obter as expressões de (31) a (40), estas expressões são válidas para ambas as conexões. A expressão é deduzida para uma fase somente, porém, o mesmo resultado é estendido às demais fases.

\subsubsection{Expressões generalizadas para $V_{c a 1}>0$ e $V_{b c 3}$ $>0$ :}

Aplicando a lei dos senos aos triângulos formados pelas amplitudes das tensões secundárias e primárias, a partir do diagrama da figura 11 (a), as expressões (31) e (32) são obtidas. 


$$
\begin{aligned}
& \frac{V_{x}}{\operatorname{sen}\left(30^{\circ}+\Psi\right)}=\frac{V_{a}}{\operatorname{sen}\left(150^{\circ}-\Psi-\alpha\right)}=\frac{V_{c a 1}}{\operatorname{sen}(\alpha)} \\
& \frac{V_{x}}{\operatorname{sen}\left(90^{\circ}+\Psi+\theta\right)}=\frac{V_{R 1}}{\operatorname{sen}\left(90^{\circ}-\Psi-\alpha\right)}=\frac{-V_{b c 3}}{\operatorname{sen}(\alpha-\theta)}
\end{aligned}
$$

\subsubsection{Expressões generalizadas para $V_{c a 1}>0$ e $V_{b c 3}$ $<0$ :}

A partir da figura 11 (b) se obtém as expressões (33) e (34):

$$
\begin{aligned}
& \frac{V_{x}}{\operatorname{sen}\left(30^{\circ}+\Psi\right)}=\frac{V_{a}}{\operatorname{sen}\left(150^{\circ}-\Psi-\alpha\right)}=\frac{V_{c a 1}}{\operatorname{sen}(\alpha)} \\
& \frac{V_{x}}{\operatorname{sen}\left(90^{\circ}-\Psi-\theta\right)}=\frac{V_{R 1}}{\operatorname{sen}\left(90^{\circ}+\Psi+\alpha\right)}=\frac{-V_{b c 3}}{\operatorname{sen}(\theta-\alpha)}
\end{aligned}
$$

\subsubsection{Expressões generalizadas para $V_{c a 1}<0$ e $V_{b c 3}$ $<0$ :}

Finalmente, a partir da figura 11 (c), as expressões (35) e (36) podem ser escritas.

$$
\begin{aligned}
& \frac{V_{x}}{\operatorname{sen}\left(150^{\circ}-\Psi\right)}=\frac{V_{a}}{\operatorname{sen}\left(30^{\circ}+\Psi+\alpha\right)}=\frac{-V_{c a 1}}{\operatorname{sen}(-\alpha)} \\
& \frac{V_{x}}{\operatorname{sen}\left(90^{\circ}-\Psi-\theta\right)}=\frac{V_{R 1}}{\operatorname{sen}\left(90^{\circ}+\Psi+\alpha\right)}=\frac{-V_{b c 3}}{\operatorname{sen}(\theta-\alpha)}
\end{aligned}
$$

\subsection{Expressão final que representa as topologias Estrela e Delta}

Observa-se das expressões de (31) a (36) que todas elas apresentam os mesmos parâmetros, tensões secundárias $V_{c a 1}$ e $V_{b c 3}$, tensão auxiliar $V_{x}$, tensão de fase primária $V_{a}$, tensão resultante no secundário do autotransformador $V_{R 1}$, o ângulo $\theta$ (que determina se o conversor é de 12 ou 18 pulsos), $\Psi$ (que indica o tipo de conexão, Estrela ou Delta) e $\alpha$ (ângulo auxiliar). Rearranjando as equações (31) a (36) se obtém a expressão (37), esta é a expressão final generalizada, foco principal deste trabalho.

$$
\begin{aligned}
& V_{a} \frac{\operatorname{sen}\left(30^{\circ}+\Psi\right)}{\operatorname{sen}\left(150^{\circ}-\Psi-\alpha\right)}=V_{c a 1} \frac{\operatorname{sen}\left(30^{\circ}+\Psi\right)}{\operatorname{sen}(\alpha)}= \\
& \quad=V_{R 1} \frac{\operatorname{sen}\left(90^{\circ}+\Psi+\theta\right)}{\operatorname{sen}\left(90^{\circ}-\Psi-\alpha\right)}=V_{b c 3} \frac{\operatorname{sen}\left(90^{\circ}+\Psi+\theta\right)}{\operatorname{sen}(\alpha-\theta)}
\end{aligned}
$$

Os valores eficazes de $V_{R 1}, V_{c a 1}$ e $V_{b c 3}$ podem ser facilmente calculados através das expressões (38), (39) e (40), respectivamente. Essas expressões podem ser solucionadas facilmente.

$$
\begin{gathered}
V_{R 1}=V_{a} \cdot \frac{\operatorname{sen}\left(30^{\circ}+\Psi\right)}{\operatorname{sen}\left(150^{\circ}-\Psi-\alpha\right)} \cdot \frac{\operatorname{sen}\left(90^{\circ}-\Psi-\alpha\right)}{\operatorname{sen}\left(90^{\circ}+\Psi+\theta\right)} \\
V_{c a 1}=V_{a} \cdot \frac{\operatorname{sen}\left(30^{\circ}+\Psi\right)}{\operatorname{sen}\left(150^{\circ}-\Psi-\alpha\right)} \cdot \frac{\operatorname{sen}(\alpha)}{\operatorname{sen}\left(30^{\circ}+\Psi\right)} \\
V_{b c 3}=V_{a} \cdot \frac{\operatorname{sen}\left(30^{\circ}+\Psi\right)}{\operatorname{sen}\left(150^{\circ}-\Psi-\alpha\right)} \cdot \frac{\operatorname{sen}(\alpha-\theta)}{\operatorname{sen}\left(90^{\circ}+\Psi+\theta\right)}
\end{gathered}
$$
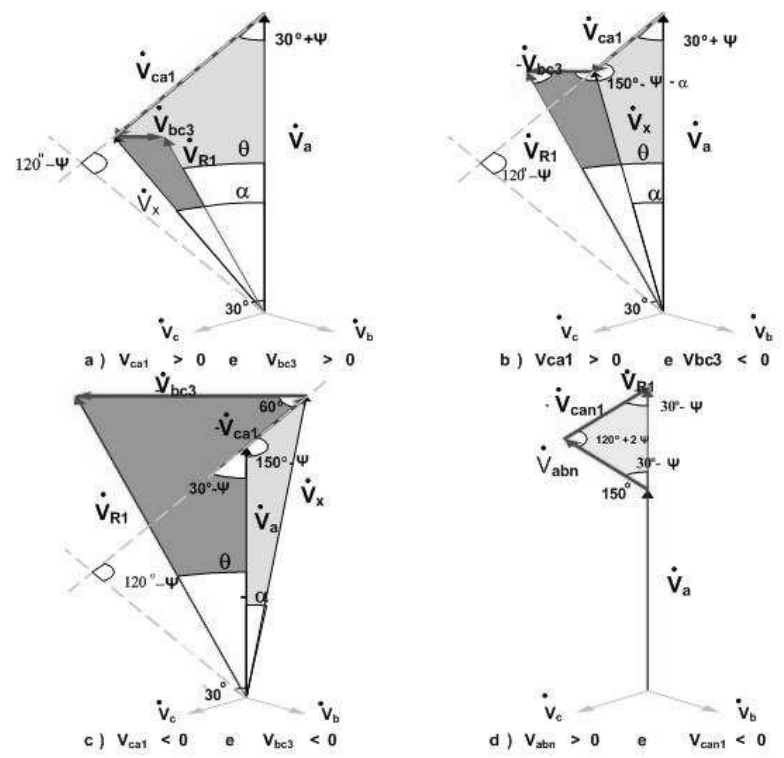

Figura 11: Diagramas fasoriais generalizados.

\subsection{Enrolamentos adicionais para 0 conversor de 18 pulsos}

As equações (41) e (42) definem as tensões através dos enrolamentos secundários adicionais. Nota-se que, para a conexão Estrela (onde $\Psi=30^{\circ}$ ), a tensão $V_{a b n 1}$ é nula. Este 
fato ocorre porque este enrolamento adicional é necessário apenas para as conexões Delta-diferenciais generalizadas.

$$
\begin{gathered}
V_{a b n}=\frac{\left(V_{R 1}-V_{a}\right)}{2 \cdot \cos \left(30^{\circ}+\Psi\right)} \\
V_{a b n 1}=\frac{\left(V_{R 1}-V_{a}\right)}{2 \cdot \cos \left(30^{\circ}+\Psi\right)} \cdot \cos (3 \cdot \Psi)
\end{gathered}
$$

\subsection{Relações de espiras}

As equações de (27) à (29) podem ser reescritas com base nos diagramas fasoriais generalizados, obtendo-se as equações de (43) à (45), válidas para as ligações Delta e Estrela. A relação de espiras $K_{a}$, é calculada através da equação (43).

$$
K_{a}=\frac{N_{a b n}}{N_{a b}}=\frac{V_{a b n}}{V_{a b}} \cdot \tan \left(45^{\circ}+\frac{\Psi}{2}\right)
$$

A relação de espiras $K_{b}$ é definida na equação (44).

$$
K_{b}=\frac{N_{c a 1}}{N_{c a}}=\frac{V_{c a 1}}{V_{c a}} \cdot \tan \left(45^{\circ}+\frac{\Psi}{2}\right)
$$

A relação de espiras $K_{c}$ é definida pela equação (45).

$$
K_{c}=\frac{N_{b c 3}}{N_{b c}}=\frac{V_{b c 3}}{V_{b c}} \cdot \tan \left(45^{\circ}+\frac{\Psi}{2}\right)
$$

Todas as curvas apresentadas nas figuras 6 e 7 para a conexão Estrela de autotransformador e as figuras 11 e 12 para a conexão Delta são válidas. $\mathrm{O}$ ângulo $\alpha$ pode ser calculado através da equação (46), conhecendo-se $V_{a}, V_{R 1}, \theta$ e $\Psi$. Aplicando o valor de nas equações (39) e (40), as tensões secundárias $V_{c a 1}$ e $V_{b c 3}$ são determinadas.

\section{EXEMPLO DE PROJETO RESULTADOS EXPERIMENTAIS}

\subsection{Exemplo de projeto}

A tabela 1 resume os parâmetros para o protótipo de um conversor de 18 pulsos com conexão Delta-diferencial com potência de $6,2 \mathrm{~kW}$. A tensão $V_{a}$ é a tensão eficaz de fase na rede, a tensão $V_{R 1}$ é a tensão de fase resultante no secundário do autotransformador, esta tensão é escolhida de forma a se obter uma tensão média na carga $\left(V_{L}\right)$ de $400 \mathrm{~V}$ (relembrando que para um retificador de seis pulsos em ponte $V_{L}$ corresponde a 2,34 vezes o valor de $V_{R 1}$ ). A Figura 12 apresenta o circuito do protótipo implementado.

Como o objetivo do trabalho é propor um método de projeto para conversores com conexões diferenciais de
Tabela 1: Parâmetros para o protótipo.

\begin{tabular}{cc}
\hline$V_{a}$ tensão eficaz de fase na entrada & $127 \mathrm{~V}$ \\
$V_{R 1}$ tensão eficaz de fase na saída & $171 \mathrm{~V}$ \\
$V_{L}$ tensão média retificada & $400 \mathrm{~V}$ \\
Número de pulsos & $18\left(\theta=20^{\circ}\right)$ \\
Topologia do autotransformador & Delta $\left(\Psi=0^{\circ}\right)$ \\
Pontes retificadoras & 3 três pontes independentes \\
\hline
\end{tabular}

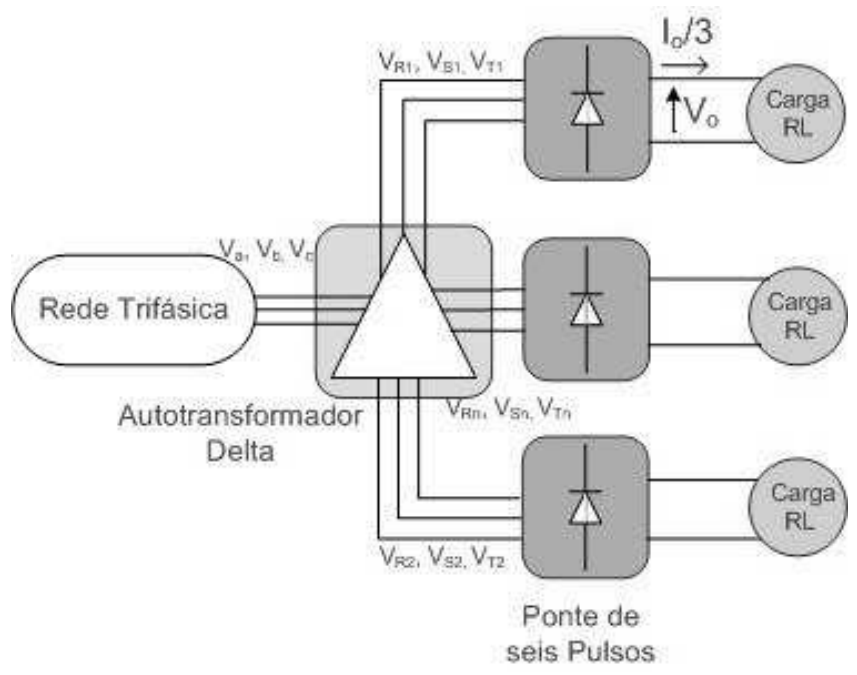

Figura 12: Circuito esquemático do protótipo implementado.

autotransformador e não especificar uma única aplicação para o conversor, o protótipo apresentado na figura 12 tem suas saídas com cargas independentes. Porém estes conversores podem ser utilizados para alimentar inversores, sistemas ininterruptos de energia (UPS), fontes para telecomunicações, entre outras, basta conectar as pontes em paralelo através de indutores de interfase (IPT), indispensáveis a este tipo de conexão. Para os testes experimentais, o valor da carga resistivo-indutiva é de $80 \Omega$ e $500 \mathrm{mH}$, para carga plena. A Figura 13 apresenta um algoritmo fácil e rápido que descreve o procedimento de projeto para conversores de 12 ou 18 pulsos com conexões Estrela ou Delta diferenciais. Aplicando os valores de $V_{a} \mathrm{e}$ $V_{R 1}$ em (46) tem-se o ângulo $\alpha=-6,892^{\circ}$ que aplicado às expressões (39) a (42), permite determinar $V_{c a 1}, V_{b c 3}, V_{a b n}$ e $V_{a b n 1}$. A tensão sobre os enrolamentos primários, $N_{a b}, N_{b c}$ e $N_{c a}$ é de 220 V. Resolvendo a expressão (39) e estendendo o resultado para todos os enrolamentos similares, a tensão sobre os enrolamentos $N_{a b 1}, N_{a b 2}, N_{b c 1}, N_{b c 2}, N_{c a 1}$ e $N_{c a 2}$ é de $-38,9 \mathrm{~V}$. A expressão (40) apresenta o cálculo para a tensão sobre os enrolamentos secundários $N_{a b 3}, N_{a b 4}, N_{b c 3}$, $N_{b c 4}, N_{c a 3}$ e $N_{c a 4}$, cujo valor é de $-77,94 \mathrm{~V}$. Finalmente, das expressões (41) e (42) obtém-se as tensões sobre os 


$$
\alpha=-\Psi-\operatorname{arctg}\left[\frac{\sqrt{3}}{3} \cdot \frac{V_{R 1} \cdot \cos (\Psi+\theta)-V_{a} \cdot \cos (\Psi)-\sqrt{3} V_{a} \operatorname{sen}(\Psi)}{\left(V_{R 1} \cdot \cos (\Psi+\theta)\right)}\right]
$$

enrolamentos $N_{a b n}, N_{a b n 1}, N_{b c n}, N_{b c n 1}, N_{c a n}$ e $N_{c a n 1}$, que são iguais a 25,4 V. Com os valores de $V_{a b}, V_{a b n}, V_{c a 1}$ e $V_{b c 3}$ obtém-se os valores das relações de espira através das equações (43) a (45): $K_{a}=8,672, K_{b}=-5,665 \mathrm{e}$ $K_{c}=-2,825$ (o valor negativo indica que os enrolamentos tem polaridades opostas aos enrolamentos primários, que formam a referência). A partir deste ponto, conhecendo as relações de espiras entre os enrolamentos, o procedimento de projeto é exatamente o mesmo aplicado a transformadores convencionais.

\subsection{Resultados experimentais}

A Tabela 2 apresenta detalhes do projeto físico do autotransformador, como número de espiras, lâminas utilizadas, peso, etc.

Tabela 2: Detalhe dos enrolamentos e núcleo.

\begin{tabular}{ll}
\hline Enrolamentos/Núcleo & Características \\
\hline \hline$N_{a b}$ & 338 espiras, 2,65 A, 17 AWG \\
\hline$N_{a b 1}=N_{a b 2}$ & 60 espiras, 4,08 A, 15 AWG \\
\hline$N_{a b 3}=N_{a b 4}$ & 120 espiras, 4,08 A, 15 AWG \\
\hline$N_{a b n}=N_{a b n 1}$ & 39 espiras, 4,08 A, 15 AWG \\
\hline \hline Perdas no núcleo, 60 Hz & $2,3 \mathrm{~W} / \mathrm{kg}$ \\
\hline Lâminas Aço/ Silício & E230, 0.54 mm espessura, \\
& Grãos não - orientados \\
\hline 'E'Laminação & Comprimento= 25 cm, \\
& Largura $=17,5 \mathrm{~cm}$ \\
\hline 'I’Laminação & Comprimento = 25 cm, Largura \\
& $=5 \mathrm{~cm}$ \\
\hline Área da seção transversal & $26 \mathrm{~cm}^{2}$ \\
\hline Peso total (enrolamentos, & $17 \mathrm{~kg}$ \\
núcleo, pontes e dissipador) & \\
\hline \hline
\end{tabular}

A Figura 14 (a) apresenta o protótipo desenvolvido, com pontes retificadoras e dissipador térmico. A figura 14 (b) apresenta as tensões para uma fase dos três sistemas secundários gerados. A tensão $V_{R S n}$ está em fase com a tensão nos enrolamentos primários (tensão de linha na rede), $V_{R S 1}$ está adiantada com relação a referência e a tensão $V_{R S 2}$ está em atraso com relação a referência. A defasagem de $\pm 20^{\circ}$, necessária para o conversor de 18 pulsos, é confirmada através do ensaio do protótipo. O autotransformador apresenta regulação de tensão, relação entre as tensões de saída com máxima carga e a vazio, de $2,8 \%$.

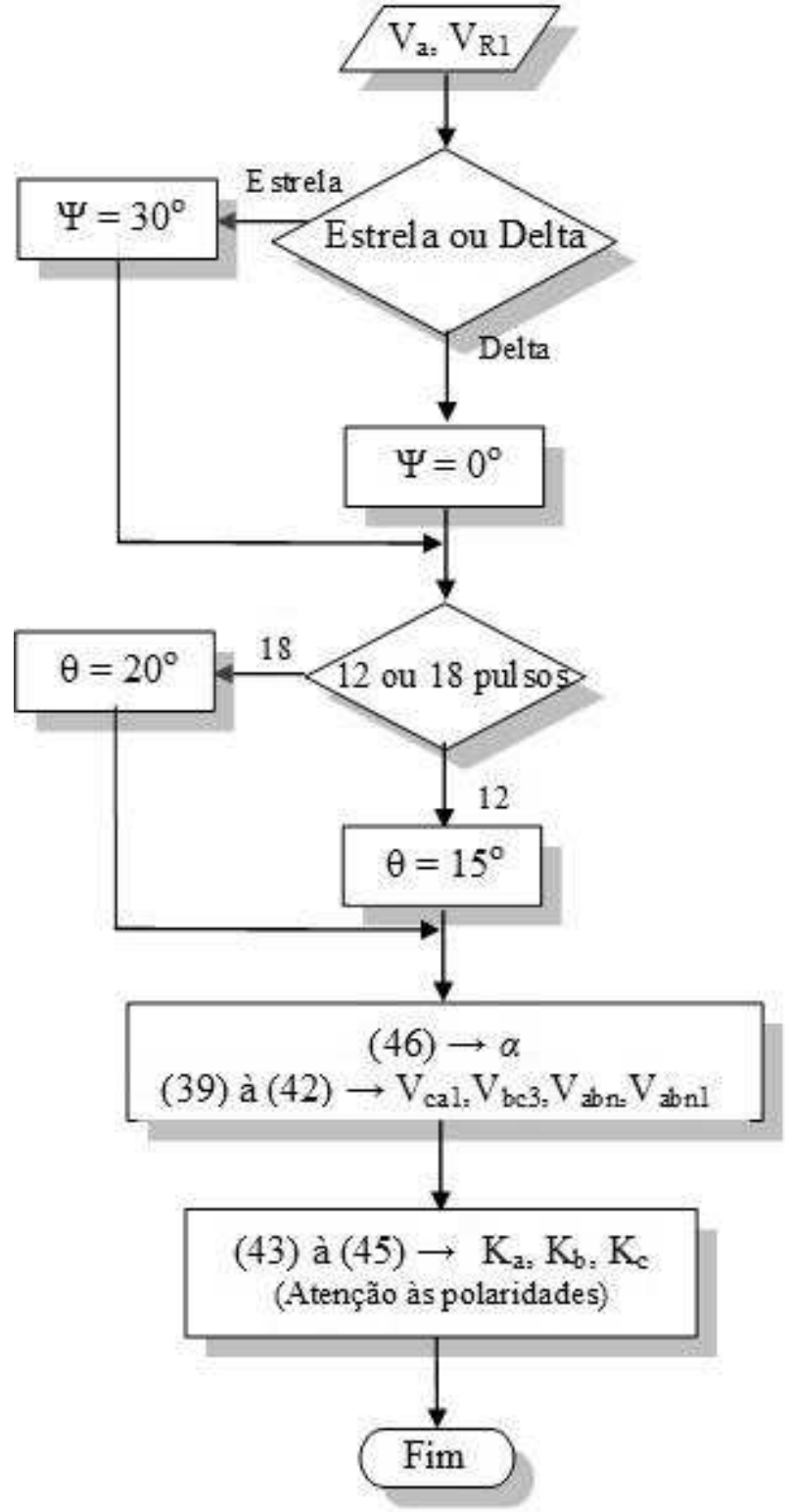

Figura 13: Algoritmo para projeto de conversores com conexões Estrela ou Delta de 12 ou 18 pulsos.

A Figura 15 (a) apresenta as correntes na rede e a tensão em uma das fases da rede. Observa-se que as correntes estão em fase com as respectivas tensões da rede e são equilibradas. Detalhes da Figura 15 (a) são apresentados na Figura 15 (b). Observa-se que as correntes na rede possuem mesma amplitude e estão defasadas de $120^{\circ}$ entre si. Há, 


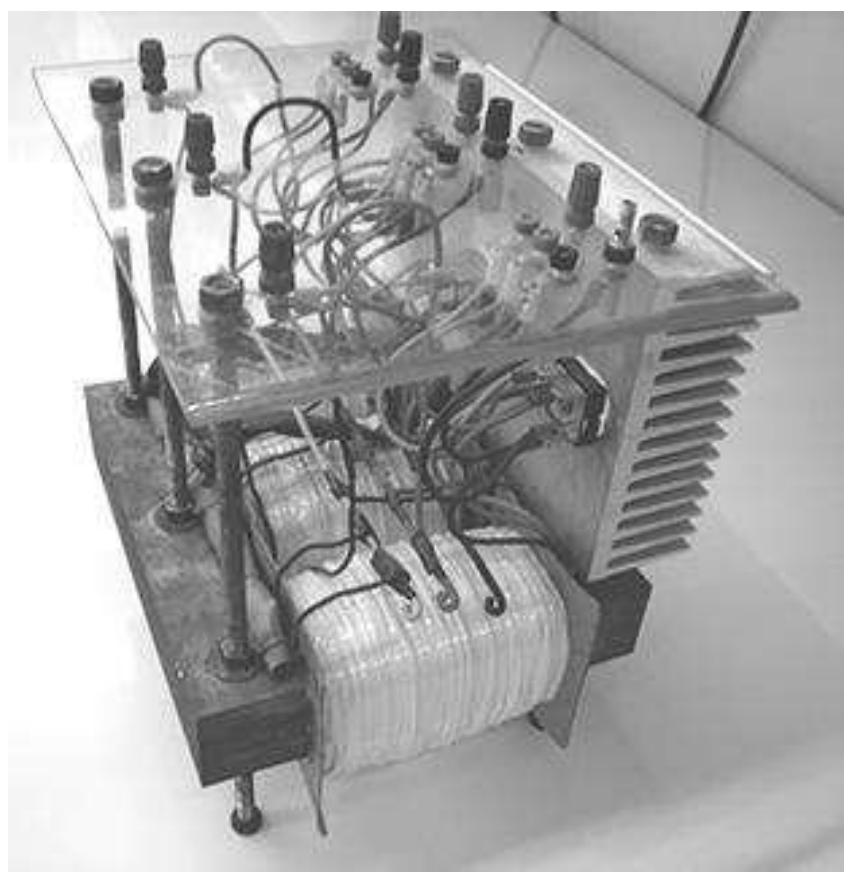

(a)

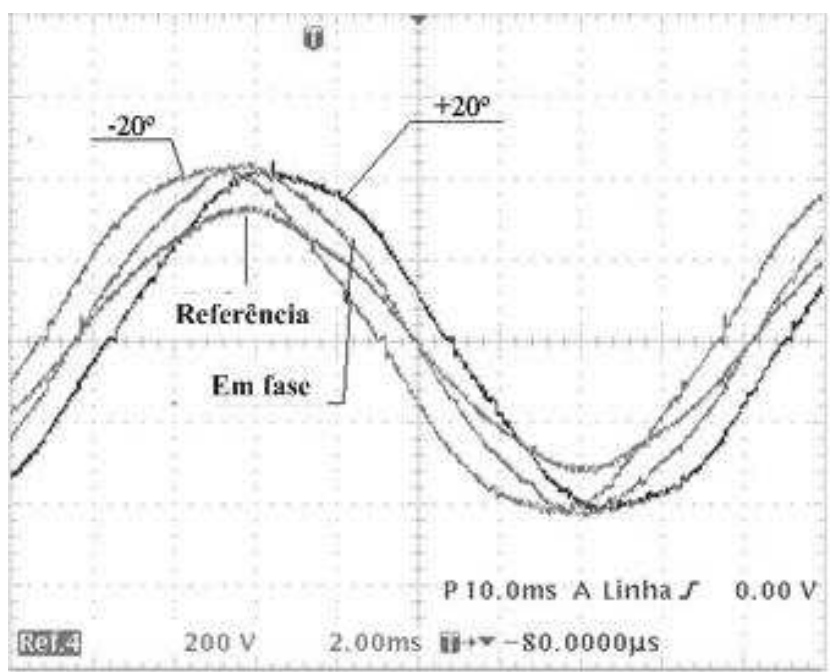

(b)

Figura 14: Foto do protótipo implementado (a) e defasagem angular entre os secundários (b).

portanto, bom equilíbrio entre as correntes. Nas figuras 15 (a) e 15 (b) a corrente está na escala de $10 \mathrm{mV} / \mathrm{A}$. O fator de deslocamento para este conversor é próximo da unidade $(0,995)$, pois o ângulo de deslocamento é pequeno $\left(6^{\circ}\right)$, assim, as correntes da rede estão praticamente em fase com as tensões.

A Figura 15 (c) apresenta o espectro harmônico para a corrente na rede, em comparação com a norma IEC

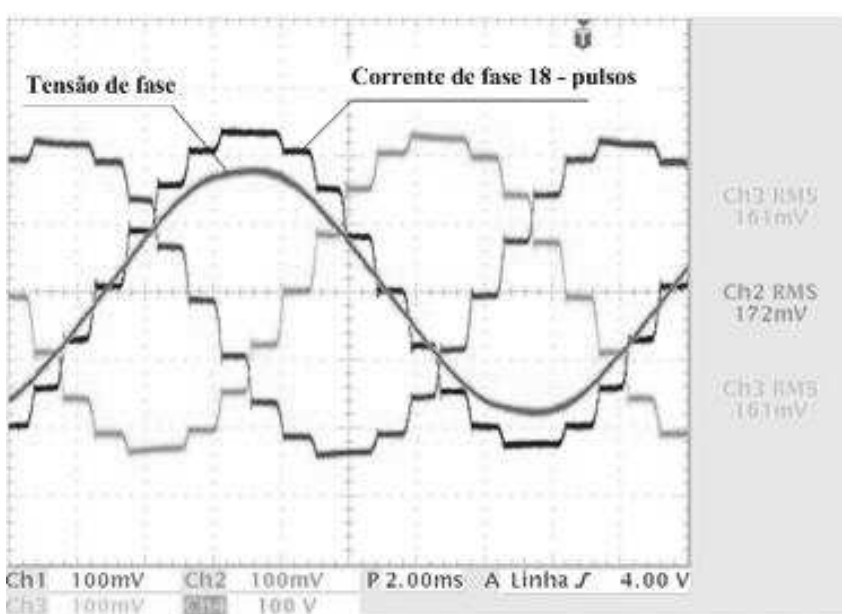

(a)

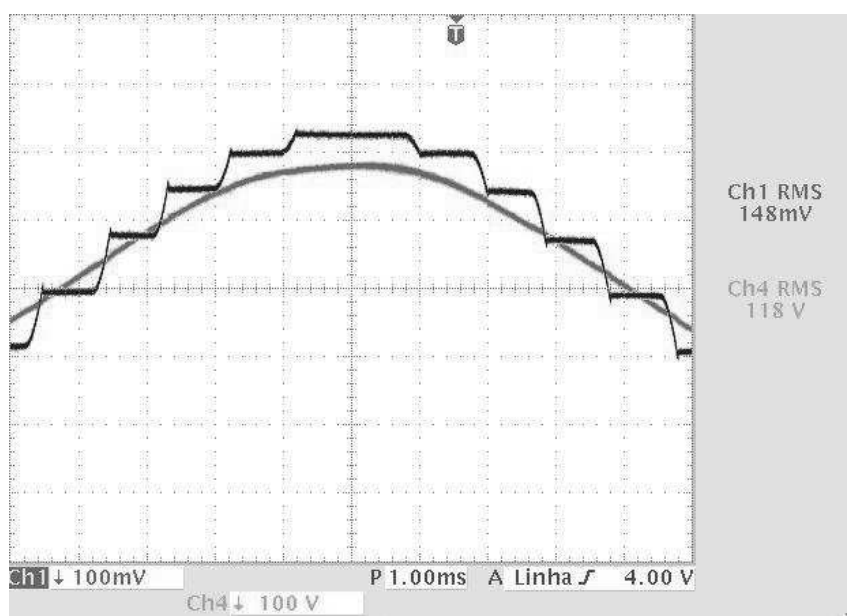

(b)

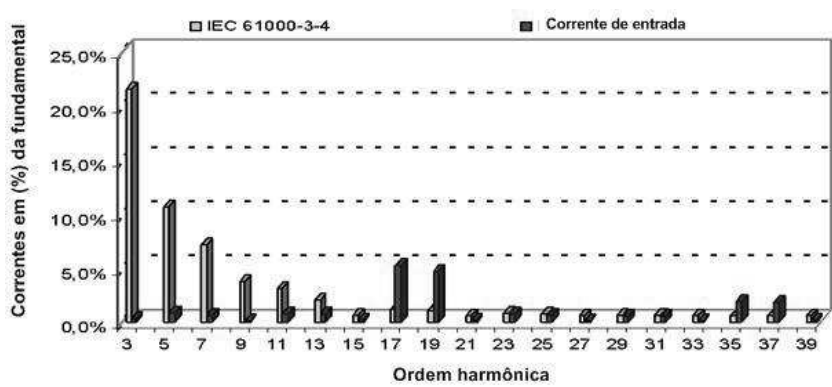

(c)

Figura 15: Corrente na rede (a), detalhe da corrente em fase com sua respectiva tensão (b) e espectro harmônico de corrente(c).

61000-3-4. Como se espera para este conversor, as amplitudes harmônicas mais significativas ocorrem para a $17^{a}, 19^{a}, 35^{a}$ e $37^{a}$ ordens harmônicas. A $D H T_{i}$ é $7,84 \%$ e o FP de 0,992. Comparando o espectro harmônico com a norma IEC 61000-3-4 (para correntes maiores que 16A por 
fase), é visível que o conversor atende até a $15^{a}$ harmônica. Assim, se for de interesse, filtros podem ser introduzidos a fim de eliminar componentes de mais alta freqüência. Exatamente por serem projetados para frequiências acima de $1 \mathrm{kHz}$ é esperado que o volume e peso destes filtros sejam baixos. A taxa kVA, relação entre a potência processada pelo núcleo magnético (kVA) e a potência que é requerida pela carga $(\mathrm{kW})$, é de 43,6\%. Assim, o núcleo magnético processa apenas uma parte da potência entregue a carga, possibilitanto redução no peso e volume do autotransformador.

A Figura 16 apresenta a curva de rendimento para o conversor implementado. Mesmo para baixa carga o rendimento é elevado. A curva mostra também que as perdas são quase constantes ao longo de toda a faixa de potência. Em plena carga o rendimento é de $97 \%$ e as perdas totais medidas são de $180 \mathrm{~W}$, incluindo perdas no núcleo magnético, enrolamentos, perdas ôhmicas e perdas nas pontes retificadoras. As pontes utilizadas (SKD 25/16) são responsáveis por $61,1 \%$ das perdas totais.

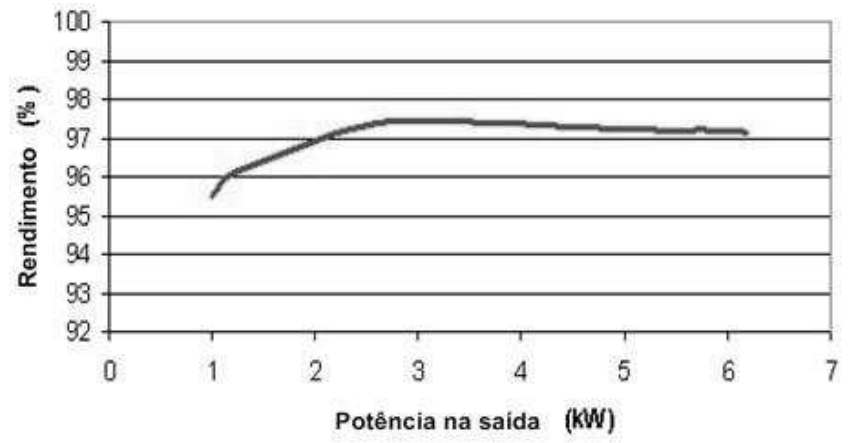

Figura 16: Rendimento.

\section{CONCLUSÕES}

Este trabalho apresenta o desenvolvimento matemático e fasorial para obtenção de um conjunto de expressões genéricas que descreve todas as conexões diferenciais de autotransformador, para as topologias Estrela e Delta, utilizados em conversores de 12 e 18 pulsos. A análise apresentada neste trabalho é de grande importância, pois torna simples o projeto de conversores multipulsos com conexões diferenciais de transformador, inclusive para aqueles pouco familiarizados nesta área de pesquisa.

O procedimento de projeto é descrito através de um fluxograma que apresenta um método rápido e fácil para o cálculo das relações de espiras e determinação das polaridades para todos os enrolamentos secundários. Considerando as expressões obtidas, curvas foram traçadas para facilitar ainda mais a obtenção das relações de espiras. Essas curvas ou ábacos fornecem diretamente as relações de espiras entre enrolamentos e indica a polaridade para se ter uma determinada tensão resultante $\left(V_{R 1}\right.$, por exemplo), em função da tensão da rede de entrada. Percebe-se um comportamento linear dessas curvas e uma possibilidade de se projetar conversores tanto abaixadores quanto elevadores de tensão.

O trabalho foi validado através da construção e ensaio do protótipo de um conversor de 18 pulsos com topologia Delta-diferencial, com potência de $6,2 \mathrm{~kW}$ e tensão média na carga de $400 \mathrm{~V}$. Neste projeto, a potência processada pelo núcleo do autotransformador foi de $43,6 \%$ da potência nominal da carga, ou seja, 2,7 kW. Através dos ensaios observou-se que o conversor (autotransformador e pontes retificadoras) atendeu às expectativas apresentando baixa $D H T_{i}$, em torno de $7,84 \%$ e, consequientemente, elevado FP $(0,992)$. Além disso, apresentou um rendimento superior a $97 \%$ e peso total de $17 \mathrm{~kg}$.

\section{AGRADECIMENTOS}

Os autores agradecem a CAPES e a FAPESP pelo apoio financeiro ao projeto. Este trabalho é dedicado à memória do nosso amigo e pesquisador Vinicius Assad Gonçalves.

\section{REFERÊNCIA BIBLIOGRÁFICA}

Burgos, R. P., A. Uan-Zo-li, F. Lacaux, A. Roshan, F. Wang, D. Boroyevich (2005). New Step-Up and Step-Down 18-Pulse Direct Asymmetric Autotransformer Rectifier Units. in Proc. IEEE Power Electronics Specialists Conf., pp. 1149-1155.

Chen, Z., J. M. Guerrero, F. Blaabjerg (2009). A Review of the State of the Art of Power Electronics for Wind Turbines. IEEE Trans. on Power Electronics, Vol. 24, pp. 1859-1875.

Freitas, L. C. G. M., G. Simões, C.A. Canesin, L.C. Freitas (2006). Programmable PFC Based Hybrid Multipulse Rectifer for Ultra Clean Power Application. IEEE Trans. on Power Electronics, Vol. 21, pp. 959-966.

Gonçalves V. A., F. J. Seixas (2006a). Generalization of the Y - Differential Autotransformer. Elsevier: Eletric Power System Research, pp. 271- 394.

Gonçalves V. A., F. J. Seixas. (2006b). Conexões Y e Delta Diferencias - Generalização para Retificadores de 12 e 18 pulsos. CBA (Congresso Brasilero de Automática), pp. $2626-2631$.

Gonçalves., V. A. (2001). Redução de Harmônicos de Corrente em Retificadores de Multiplos Pulsos Generalização das Conexões Diferenciai. Dissertação 
de Mestrado. UNESP - Faculdade de Engenharia de Ilha Solterira, pp. 177.

Kazmierkowski, M., P. R. Krishnan, F. Blaabjerg (2009). Control in Power Electronics. San Diego, CA: Elsevier, Chap. 11, 12 and 13.

Pomilio, J. A., L. Rossetto, P. Tomasin, (1997). Performance Improvement of Soft-Switched PWM Rectifiers with Inductive Load. IEEE Trans. on Power Electronics, Vol. 12, pp. 153-160.

Paice D. A. (1996). Power Electronic Converter Harmonic Multipulse Methods for Clean Power. New York: IEEE Press.

Schaefer, Rectifier Circuits (1965). Theory and Design. Norwalk: John Wiley and Sons.

Seixas F. J. M., V. A. Gonçalves (2007). Generalização das Conexões Delta - Diferenciais para Retificadores de 12 e 18 pulsos. Eletrônica de Potência, Vol. 12, n ${ }^{o}$. 1; pp. $35-42$

Seixas F. J. M., I. Barbi (2004). A 12 kW Three-Phase Low THD Rectifier with High-Frequency Isolation and Regulated DC Output. IEEE Trans. on Power Electronics, Vol. 19, pp. 371-377.

Seixas, F. J. M., I. Barbi (2001a). A New Three-Phase Low THD Power Supply with High-Frequency Isolation and 60V/200A Regulated DC Output. in Proc. IEEE Power Electronics Specialists Conf., pp.1629-1634.

Seixas F. J. M. (2001b). Conversores CA - CC de $12 \mathrm{~kW}$ com elevado Fator de Potência utilizando Autotransformador com Conexão Diferencial de Multipulsos, Tese (Doutorado). Universidade Federal de Santa Catarina, pp. 205.

Singh, B., S. Gairola, B. N. Singh, A. Chandra, K. Al-Haddad, K. (2008). Multipulse AC-DC Converters for Improving Power Quality: A Review. IEEE Trans. on Power Electronics, Vol. 23, pp. 260-268. 